\title{
Article
}

\section{Inhibition of BET Proteins during Adolescence Affects Prefrontal Cortical Development: Relevance to Schizophrenia}

\author{
Wiktor Bilecki, Agnieszka Wawrzczak-Bargieła, Iwona Majcher-Maślanka, Magdalena Chmelova and \\ Marzena Maćkowiak *iD
}

check for

updates

Citation: Bilecki, W.;

Wawrzczak-Bargieła, A.;

Majcher-Maślanka, I.; Chmelova, M.;

Maćkowiak, M. Inhibition of BET

Proteins during Adolescence Affects

Prefrontal Cortical Development:

Relevance to Schizophrenia. Int. J.

Mol. Sci. 2021, 22, 8710. https://

doi.org/10.3390/ijms22168710

Academic Editor: Juan

F. Lopez-Gimenez

Received: 9 July 2021

Accepted: 9 August 2021

Published: 13 August 2021

Publisher's Note: MDPI stays neutral with regard to jurisdictional claims in published maps and institutional affiliations.

Copyright: (c) 2021 by the authors. Licensee MDPI, Basel, Switzerland. This article is an open access article distributed under the terms and conditions of the Creative Commons Attribution (CC BY) license (https:// creativecommons.org/licenses/by/ $4.0 /)$

\author{
Laboratory of Pharmacology and Brain Biostructure, Department of Pharmacology, Maj Institute of \\ Pharmacology, Polish Academy of Sciences, Smętna Str. 12, 31-343 Kraków, Poland; \\ bilecki@if-pan.krakow.pl (W.B.); bargiela@if-pan.krakow.pl (A.W.-B.); majcher@if-pan.krakow.pl (I.M.-M.); \\ chmelova@if-pan.krakow.pl (M.C.) \\ * Correspondence: mackow@if-pan.krakow.pl; Tel.: +48-12-6623-262; Fax: +48-12-637-4500
}

\begin{abstract}
Background: The present study investigated the role of proteins from the bromodomain and extra-terminal (BET) family in schizophrenia-like abnormalities in a neurodevelopmental model of schizophrenia induced by prenatal methylazoxymethanol (MAM) administration (MAM-E17). Methods: An inhibitor of BET proteins, JQ1, was administered during adolescence on postnatal days (P) 23-P29, and behavioural responses (sensorimotor gating, recognition memory) and prefrontal cortical (mPFC) function (long-term potentiation (LTP), molecular and proteomic analyses) studies were performed in adult males and females. Results: Deficits in sensorimotor gating and recognition memory were observed only in MAM-treated males. However, adolescent JQ1 treatment affected animals of both sexes in the control but not MAM-treated groups and reduced behavioural responses in both sexes. An electrophysiological study showed LTP impairments only in male MAM-treated animals, and JQ1 did not affect LTP in the MPFC. In contrast, MAM did not affect activity-dependent gene expression, but JQ1 altered gene expression in both sexes. A proteomic study revealed alterations in MAM-treated groups mainly in males, while JQ1 affected both sexes. Conclusions: MAM-induced schizophrenia-like abnormalities were observed only in males, while adolescent JQ1 treatment affected memory recognition and altered the molecular and proteomic landscape in the mPFC of both sexes. Thus, transient adolescent inhibition of the BET family might prompt permanent alterations in the mPFC.
\end{abstract}

Keywords: bromodomain; neurodevelopment; JQ1; learning and memory; cognition

\section{Introduction}

Schizophrenia is a neurodevelopmental disorder whose first symptoms are usually diagnosed in late adolescence or early adulthood [1]. However, structural and functional abnormalities observed in individuals with schizophrenia are considered the result of early-life developmental disruption, and the neuromaturational process during adolescence appears to be important for the clinical aspects of the illness (reviewed in [1,2]). The latter observation is supported by several findings indicating that environmental or pharmacological interventions during childhood or adolescence might alter the course of schizophrenia (reviewed in $[3,4]$ ). The apparent lack of consistently replicated major genetic defects and the increasing evidence of changes in gene expression after exposure to environmental risk factors suggest that epigenetic regulation might be involved in the aetiology of schizophrenia (reviewed in [5]). However, limited data related to epigenetic regulation, especially in adolescence, of alterations in the developmental trajectory of the brain predisposed genetically or environmentally to schizophrenia symptom appearance are available.

The histone acetylation pattern is one of the epigenetic modifications that might be critical to gene transcription and involved in the brain maturation process [6]. Moreover, 
previous data from our [7] and other [8] studies using animal neurodevelopmental models of schizophrenia indicated that the regulation of histone acetylation in the medial prefrontal cortex (mPFC) during early adolescence might be an important mechanism involved in the development of schizophrenia-like abnormalities in adulthood. The pattern of acetylation marks is recognised by acetyl-lysine residues such as bromodomain (epigenetic readers) functioning as a scaffold for assembly of macromolecular complexes that alter chromatin accessibility to transcription factors and allow the recruitment or activation of RNA polymerases [9]. These complexes then initiate transcriptional programmes that result in altered gene expression. Epigenetic readers of acetylation marks from the bromodomain and extra-terminal domain (BET) family have been shown to be key regulators of chromatin dynamics and the disease-associated acetylome $[9,10]$. The BET family is comprised of four proteins: BRD2, BRD3, and BRD4, which are expressed in most cells and tissues, and BRDT that is primarily localised in the testes [11]. Acetyl-lysine mimic compounds, namely, BET inhibitors (JQ1, I-BET, and MS417) with high affinity and specificity to BET bromodomains, function by blocking BET proteins from binding acetylated chromatin. Compounds regulating the function of BET proteins might alter epigenetic status and affect transcriptional activity in cells $[9,10]$.

Some evidence indicates the involvement of BET proteins in neuroplasticity regulation and memory formation, as well as their potential roles in the pathomechanisms of neurodevelopmental disorders [12-14]. Findings from animal studies showed a selective upregulation of BRD4 protein in an animal model of Fragile X Syndrome (FXS), a neurodevelopmental disorder that might cause intellectual disability or autism spectrum disorder [13]. An inhibition of BRD4 function diminished transcriptional disruption and reversed behavioural deficits observed in an FXS animal model [13]. On the other hand, pharmacological suppression of BET protein during mice adolescence led to selective repression of neuronal gene expression and development of autism-like syndrome [15]. However, BET protein involvement in pathogenesis of other neurodevelopmental disorders, i.e., schizophrenia, remains unknown. Therefore, in the present study, we investigated the role of the BET protein family in early adolescence, the period of proper neurodevelopment and emergence of schizophrenia-like abnormalities, in a neurodevelopmental animal model of schizophrenia induced by prenatal administration of an antimitotic agent, methylazoxymethanol (MAM), at embryonic day 17 (MAM-E17 model) [16]. We determined whether the administration of the BET family inhibitor JQ1 during early adolescence (postnatal day (P) 23-P29) may affect the behavioural response in adult rats and mPFC neurodevelopment. Our previous study using the MAM-E17 model showed that the chosen period is a critical window for epigenetically driven mPFC maturation and the development of behavioural and neurochemical schizophrenia-like abnormalities in adult MAM-E17 rats $[7,17,18]$. Therefore, in the present study, we analysed behavioural responses related to cognitive function: sensorimotor gating $[19,20]$ and recognition memory [21,22]. An electrophysiological study measuring long-term potentiation (LTP) was also performed to determine adult mPFC function at the cellular level. Moreover, the expression of genes in the BET family (BRD2, BRD3, and BRD4) was measured to verify an effect of adolescent BET protein inhibition on the function of the BET family in the adult mPFC. In addition, neuronal activation in the MPFC was examined by measuring the expression of immediate early genes (IEGs: c-Fos, activity-regulated cytoskeletal (Arc), and neuronal PAS domain protein 4 (Npas4)) [23] that might be regulated by BET proteins [12].

The cortical dysfunction of GABAergic transmission observed in the prefrontal cortex of individuals with schizophrenia might result from developmental abnormalities [24], which was also confirmed in our previous study showing impaired transcription of genes that are markers of the GABA system, such as glutamic acid decarboxylase (GAD)67 and parvalbumin (PV), in the adult mPFC of MAM-E17 animals $[17,25]$. Thus, we verified that the expression of GAD67 and PV genes in the adult mPFC was related to dysfunction of BET proteins during adolescence. In addition to investigating the functional and molecular aspects of adolescent inhibition of BET proteins in the adult $\mathrm{MPFC}$, we also designed a 
proteomic study to analyse the pattern of protein alterations in the adult MPFC induced by adolescent disruption of BET protein function.

The experiments were designed to assess both sexes (males and females) because of findings showing sex differences in psychiatric disorders, i.e., schizophrenia [26]. Moreover, in the MAM model of schizophrenia, different results related to the development of schizophrenia-like abnormalities are observed in males and females [27-29]. Thus, in our study, we also investigated whether adolescent inhibition of the BET family might exert a sex-dependent effect on the development of schizophrenia-like dysfunction.

\section{Results}

\subsection{Behavioural Response}

We studied recognition memory and sensorimotor gating in both males and females to determine whether adolescent BET protein inhibition affected cognitive function in MAM-E17 rats.

\subsubsection{Recognition Memory in Males}

MAM treatment did not induce any significant differences in the time spent exploring two identical objects presented during the training session (prenatal factor $\times$ object: $F(1$, $44)=3.78, p=0.06$ ), and no significant differences were observed between groups (prenatal $\times$ postnatal factors $\times$ object: $\mathrm{F}(1,44)=0.27 ; p=0.61$; Figure $1 \mathrm{~A})$.
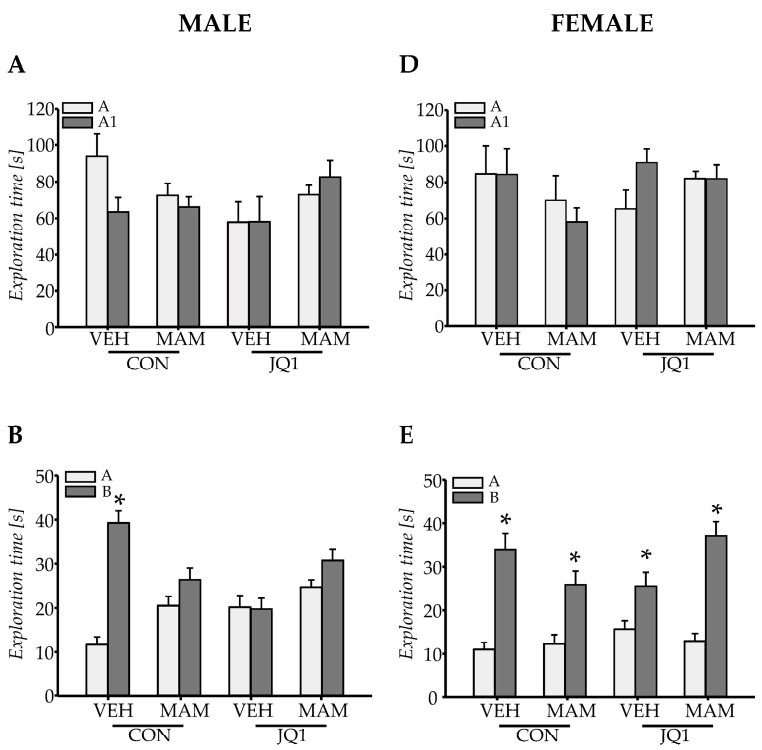

E
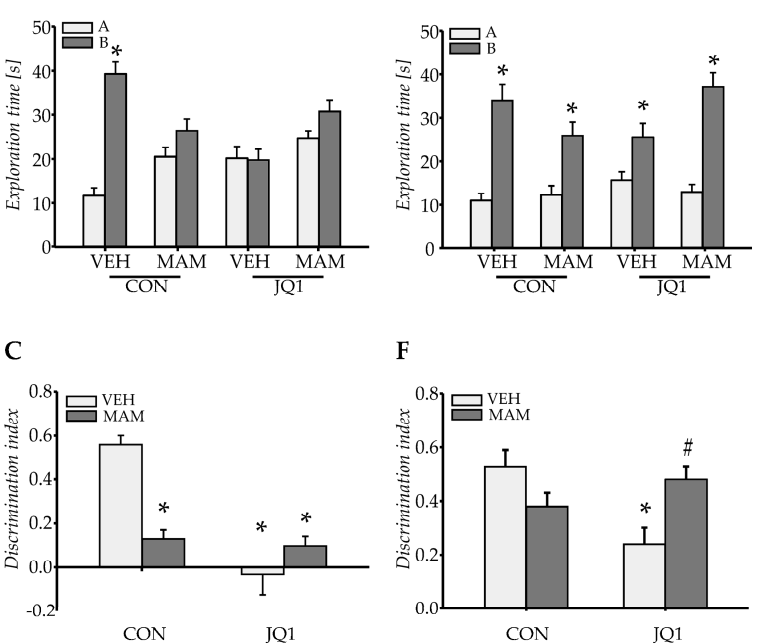

Figure 1. The effect of adolescent JQ1 administration on recognition memory in the novel object recognition test in the MAM-E17 model of schizophrenia. $(\mathbf{A}, \mathbf{D})$ Exploration time of two identical objects in the acquisition session, $(\mathbf{B}, \mathbf{E})$ exploration time of one novel and one familiar object in the retention test conducted $1 \mathrm{~h}$ following the acquisition session, $(\mathbf{C}, \mathbf{F})$ discrimination index. The rats were (males: A-C and females: D-F) exposed to JQ1 or vehicle (CON) in early adolescence (P23-P29), and the analyses were performed in rats at P60. Each data point represents the mean $\pm S E M ; n=12$ per group. $\mathbf{B}-{ }^{*}$ $p$ textless 0.05 vs. familiar object (A), C- ${ }^{*} p$ textless 0.05 vs. VEH-CON, $\# p$ textless 0.05 vs. MAM-CON (two-way repeated measures ANOVA $(\mathbf{A}, \mathbf{B}, \mathbf{D}, \mathbf{E})$ or two-way ANOVA $(\mathbf{C}, \mathbf{F})$ followed by a Tukey test). 
However, in the retention session, MAM exerted statistically significant effects on discrimination of the novel object from the familiar object (prenatal factor $\times$ object: $F(1$, $44)=14.93 ; p$ textless 0.0004$)$, and an interaction between MAM administration and adolescent JQ1 treatment was observed (prenatal $\times$ postnatal factors $\times$ object: $\mathrm{F}(1,44)=51.13$; $p$ textless 0.000001$)$. VEH-CON rats spent significantly more time exploring the novel object than the familiar object ( $p$ textless 0.003 , Figure 1B). However, MAM-CON and MAM-JQ1 rats did not discriminate between the objects and spent similar amounts of time exploring each object ( $p=0.065$, Figure 1B). Adolescent JQ1 administration affected the exploration of the novel object by rats in the VEH group, and these animals did not discriminate between the objects and spent similar amounts of time exploring each object ( $p=0.99$, Figure 1B).

A significant effect of MAM treatment on the discrimination index was observed (prenatal factor: $\mathrm{F}(1,44)=7.7 ; p$ textless 0.009 ), and a significant interaction between MAM and JQ1 was identified (prenatal $\times$ postnatal factors: $\mathrm{F}(1,44)=27.44 ; p$ textless 0.000005 ). A similar decrease in the discrimination index was observed in all groups when compared to the VEH-CON group ( $p$ textless 0.0002); however, the strongest effect was observed in the VEH-JQ1 group (Figure 1C).

\subsubsection{Recognition Memory in Females}

MAM treatment did not induce any significant differences in time spent exploring two identical objects presented during the training session (prenatal factor $\times$ object: $\mathrm{F}(1$, $44)=0.73 ; p=0.4$ ), and significant differences were not observed between groups (prenatal $\times$ postnatal factors $\times$ object: $\mathrm{F}(1,44)=0.14 ; p=0.71$; Figure 1D).

In the retention session, MAM did not affect the discrimination of the novel object from the familiar object (prenatal factor $\times$ object: $\mathrm{F}(1,44)=0.72 ; p=0.4)$; however, an interaction between MAM administration and adolescent JQ1 treatment was observed (prenatal $\times$ postnatal factors $\times$ object: $\mathrm{F}(1,44)=17.23$; $p$ textless 0.0002$)$. All groups spent significantly more time exploring the novel object than the familiar object $(p$ textless 0.0002 for the VEH-CON group, $p$ textless 0.0006 for the MAM-CON group, $p$ textless 0.02 for the VEH-JQ1 group, and $p$ textless 0.0002 for the MAM-JQ1 group; Figure 1E).

MAM also did not alter the discrimination index (prenatal factor: $F(1,44)=0.68$; $p=0.41$ ); however, an interaction between MAM administration and adolescent JQ1 treatment was observed (prenatal $\times$ postnatal factors: $\mathrm{F}(1,44)=14.69 ; p$ textless 0.0005 ). MAM alone did not change the discrimination index ( $p=0.18$ compared with the VEH-CON group). However, JQ1 decreased the discrimination index in the VEH group ( $p$ textless 0.002 compared with the VEH-CON group and $p$ textless 0.02 compared with the MAM-JQ1 group), and that effect was not observed in the MAM-JQ1 group ( $p=0.91$ compared with the VEH-CON group, Figure 1F).

The results showed a deficit in recognition memory only in the female VEH-JQ1 group.

\subsubsection{Sensorimotor Gating in Males}

The acoustic pre-pulse intensity exerted significant effects on PPI $(F(2,88)=36.76$, $p$ textless 0.0000001, Figure 2A).

The results revealed that either MAM or JQ1 administration affected recognition memory in males.

MAM treatment did not affect PPI in the adult rats (prenatal factor: $\mathrm{F}(1,44)=0.197$; $p=0.66)$, and the effect of MAM was not determined by the pre-pulse intensity used (prenatal factor $\times$ pre-pulse: $\mathrm{F}(2,88)=0.61 ; p=0.54)$. However, adolescent JQ1 administration altered PPI in adulthood (postnatal factor: $\mathrm{F}(1,44)=5.76 ; p$ textless 0.03 ), but the effect of JQ1 was not determined by the pre-pulse intensity used (postnatal factor $\times$ pre-pulse: $F(2$, $88)=0.058 ; p=0.95$ ). Moreover, a statistically significant interaction between MAM and JQ1 treatment was observed (prenatal $\times$ postnatal factors: $\mathrm{F}(1,44)=7.72 ; p$ textless 0.009 ); however, the effect did not depend on the intensity of the applied pre-pulse (prenatal $\times$ postnatal factors $\times$ pre-pulse: $\mathrm{F}(2,88)=0.44, p=0.65$, Figure $2 \mathrm{~A})$. 
A

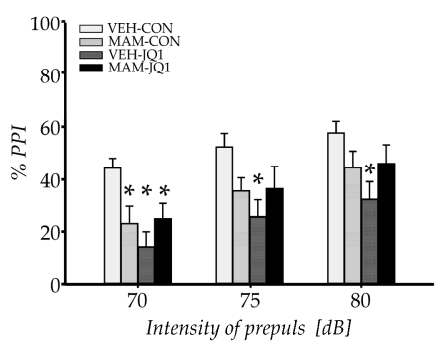

B

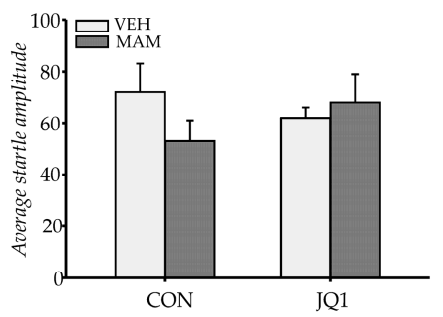

C

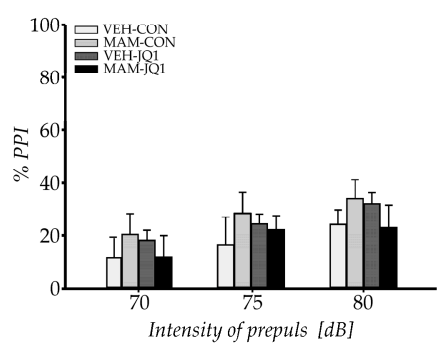

D

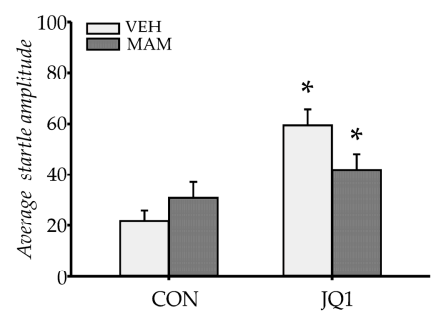

Figure 2. The effects of an adolescent JQ1 administration on sensorimotor gating (\% of pre-pulse inhibition, PPI) in the acoustic startle response test $(\mathbf{A}, \mathbf{C})$ and on the average amplitude of startle $(\mathbf{B}, \mathbf{D})$ in the MAM-E17 model of schizophrenia. The rats (males: $\mathbf{A}, \mathbf{B}$ and females: $\mathbf{C , D}$ ) were exposed to JQ1 or vehicle (CON) in early adolescence (P23-P29), and the analyses were performed in rats at P70. Each data point represents the mean $\pm \mathrm{SEM} ; \mathrm{n}=12$ per group * $p$ textless $0.05 \mathrm{vs}$. VEH-CON (two-way repeated measures ANOVA $(\mathbf{A}, \mathbf{C})$ or two-way ANOVA (B,D) followed by a Tukey test).

MAM induced a statistically significant decrease in PPI only at the lowest pre-pulse intensity used ( $p$ textless 0.04 at $70 \mathrm{~dB}$ compared with the VEH-CON group). JQ1 administration decreased PPI in the VEH group at all applied pre-pulse intensities ( $p$ textless 0.03 at $70 \mathrm{~dB}, p$ textless 0.05 at $75 \mathrm{~dB}$, and $p$ textless 0.005 at $80 \mathrm{~dB}$ for the comparison of the VEH-CON group with the appropriate group); however, JQ1 did not alter PPI in the MAM-treated group (Figure 2A).

In addition, the average startle amplitude was analysed in each experiment. Neither MAM nor JQ1 altered the average startle amplitude (prenatal $\times$ postnatal factors: $F(1$, 44) $=5.24 ; p=0.14$, Figure 2B).

The results revealed a deficit in sensorimotor gating in all analysed male groups, however the strongest effect was observed in the VEH-JQ1 group.

\subsubsection{Sensorimotor Gating in Females}

Significant effects of the acoustic pre-pulse intensity on PPI were observed F (2, $88)=18.8, p$ textless 0.0000001 , Figure $2 \mathrm{C})$.

Neither MAM (prenatal factor $\times$ pre-pulse: $\mathrm{F}(2,88)=0.62 ; p=0.54)$ nor adolescent JQ1 treatment (postnatal factor $\times$ pre-pulse: $\mathrm{F}(2,88)=0.16 ; p=0.85)$ affected PPI at all applied pre-pulse intensities. An interaction between factors was not observed (prenatal $\times$ postnatal factors $\times$ pre-pulse: $\mathrm{F}(2,88)=0.16 ; p=0.86$, Figure $2 \mathrm{C})$.

However, the average startle amplitude analysed in each experiment was affected by adolescent JQ1 treatment (postnatal factor: $\mathrm{F}(1,44) 21.53 ; p$ textless 0.00004$)$. MAM did not alter the average startle amplitude (prenatal factor: $\mathrm{F}(1,44)=0.68 ; p=0.42)$; however, an interaction between MAM and JQ1 treatment was observed (prenatal $\times$ postnatal factors: $\mathrm{F}(1,44)=6.62 ; p$ textless 0.014$)$. Adolescent administration of JQ1 increased the startle 
amplitude in both the VEH- and MAM-treated groups ( $p$ textless 0.0003 and $p$ textless 0.05 respectively, compared with the VEH-CON group, Figure 2D).

Neither MAM nor JQ1 administration affected sensorimotor gating in females, however adolescent JQ1 treatment increased the average startle amplitude in both the VEHand MAM-treated groups.

\subsection{Electrophysiological Recordings in the Adult Medial Prefrontal Cortex}

We studied long-term potentiation in both males and females to investigate whether prenatal MAM administration and/or adolescent exposure to JQ1 affects functional synaptic plasticity in the mPFC.

\subsubsection{LTP in Males}

Significant effects of tetanisation were observed (tetanisation: $\mathrm{F}(1,112)=62.98, p$ textless 0.000001). Both prenatal and postnatal treatment factors affected tetanisation of the field potential amplitude (prenatal factor $\times$ tetanisation: $\mathrm{F}(1,112)=14.29 ; p$ textless 0.0003 ) and postnatal factor $\times$ tetanisation: $F(1,112)=5.51 ; p$ textless 0.03$)$. No statistically significant interactions between the analysed factors and tetanisation were observed (prenatal $\times$ postnatal factors $\times$ tetanisation: $\mathrm{F}(1,112)=0.25, p=0.62)$ ).

An analysis of the magnitude of potentiation revealed that the MAM groups generally displayed a smaller potentiation than the VEH-CON rats ( $p$ textless 0.006 for the MAMCON group and $p$ textless 0.00005 for the MAM-JQ1 group). However, no differences in LTP were observed in VEH-JQ1 rats $(p=0.24$ compared with the VEH-CON group, Figure 3).

A B
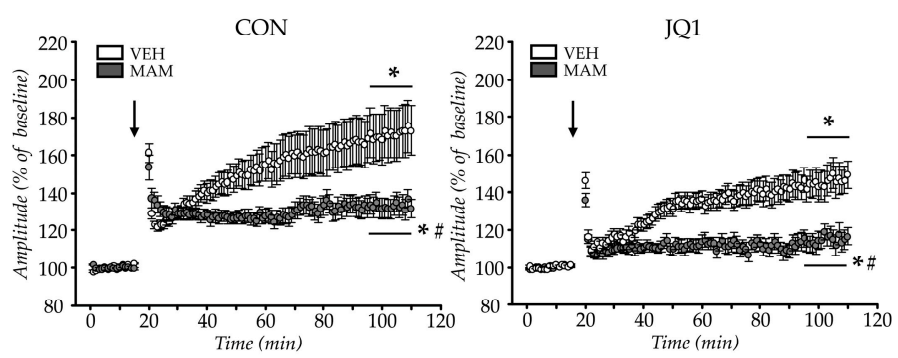

C

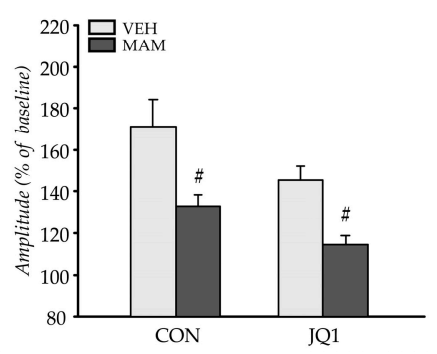

Figure 3. The effects of an adolescent JQ1 administration on long-term potentiation (LTP) in the male adult medial prefrontal cortex in the MAM-E17 model of schizophrenia. The rats were exposed to JQ1 or vehicle (CON) in early adolescence (P23-P29), and the analyses were performed in rats at P60-P110. The data are presented as group mean \pm SEM, $n=12-17$ (the number of brain sections from 6 to 9 animals). The data are expressed as percentage of the baseline amplitude values for each time point (A-C), C - the average of LTP during the last $15 \mathrm{~min}$ of recording. ${ }^{*} p$ textless 0.05 compared with the last $15 \mathrm{~min}$ of pre-tetanisation recording (for values averaged over the last $15 \mathrm{~min}$ of recording); \# $p$ textless 0.05 compared with VEH-CON animals (for values averaged over the last 15 min of recording); two-way repeated measures ANOVA $(\mathbf{A}, \mathbf{B})$ or two-way ANOVA $(\mathbf{C})$ followed by a Tukey test. Arrows indicate time of delivery of tetanic stimulation. 
The results showed a deficit in LTP in the adult mPFC of the MAM-treated males, and JQ1 treatment did not affect LTP formation in any analysed group.

\subsubsection{LTP in Females}

Significant effects of tetanisation were observed (tetanisation: $F(1,115)=69.32$; $p$ textless 0.000001$)$. Neither prenatal nor postnatal treatment factors affected tetanisation of the field potential amplitude (prenatal factor $\times$ tetanisation: $\mathrm{F}(1,115)=0.81 ; p=0.37$ and postnatal factor $\times$ tetanisation: $F(1,115)=2.19 ; p=0.14)$. Statistically significant interactions between the analysed factors and tetanisation were not observed $(\mathrm{F}(1,115)=0.26, p=0.61$, Figure 4).
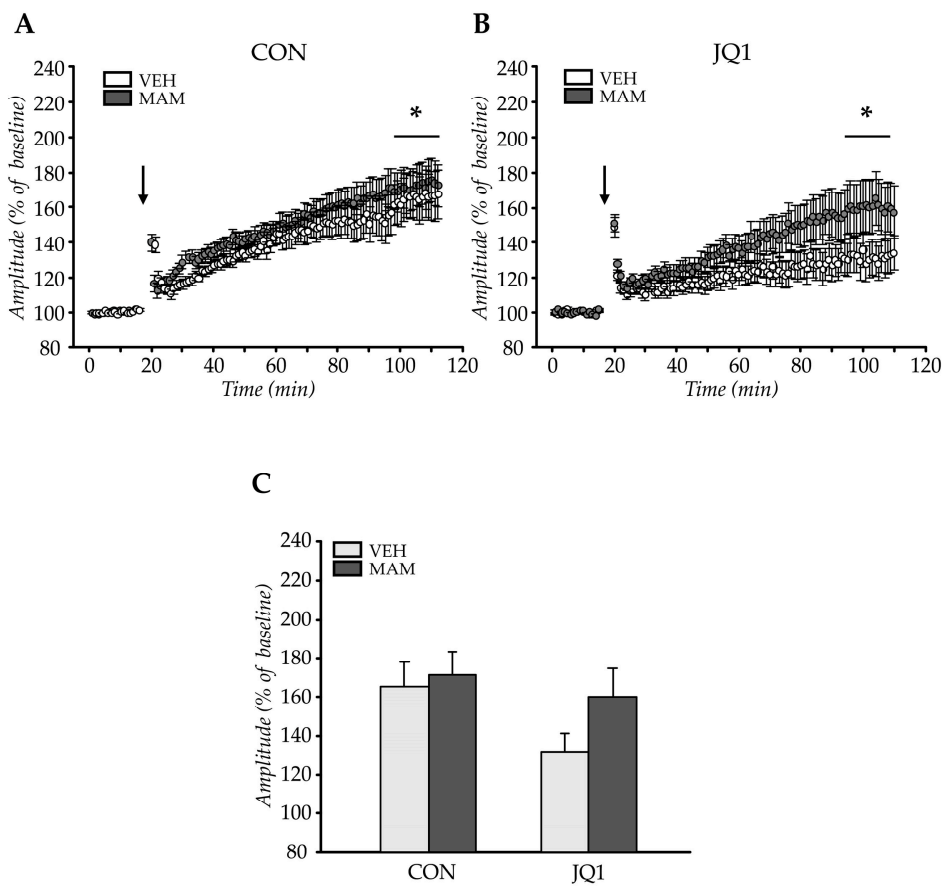

Figure 4. The effects of an adolescent JQ1 administration on long-term potentiation (LTP) in the female adult medial prefrontal cortex in the MAM-E17 model of schizophrenia. The rats were exposed to JQ1 in early adolescence (P23-P29) or vehicle (CON), and the analyses were performed in rats at P60-P110. The data are presented as group mean \pm SEM, $n=12-17$ (the number of brain sections from 6 to 9 animals). The data are expressed as percentage of the baseline amplitude values for each time point (A-C), C-the average of LTP during the last $15 \mathrm{~min}$ of recording. ${ }^{*} p$ textless 0.05 compared with the last $15 \mathrm{~min}$ of pre-tetanisation recording (for values averaged over the last $15 \mathrm{~min}$ of recording); two-way repeated measures ANOVA (A,B) or two-way ANOVA (C) followed by a Tukey test. Arrows indicate time of delivery of tetanic stimulation.

Neither prenatal MAM nor postnatal JQ1 administration affected LTP formation in the female adult mPFC.

\subsection{Molecular Studies in the Adult Medial Prefrontal Cortex}

\subsubsection{BET Family Genes}

We analysed the mRNA levels of $B R D 2, B R D 3$, and $B R D 4$ in the mPFC of both males and females to determine whether adolescent JQ1 administration affected the transcription of the BET family.

\section{- $\quad$ BRD2 expression}

Prenatal MAM administration did not alter the expression of the BRD2 mRNA in the mPFC of males (prenatal factor: $\mathrm{F}(1,20)=0.12 ; p=0.73$ ). No interaction was observed 
between the two analysed factors (prenatal $\times$ postnatal factors: $\mathrm{F}(1,20)=0.001 ; p=0.98$, Figure 5A).
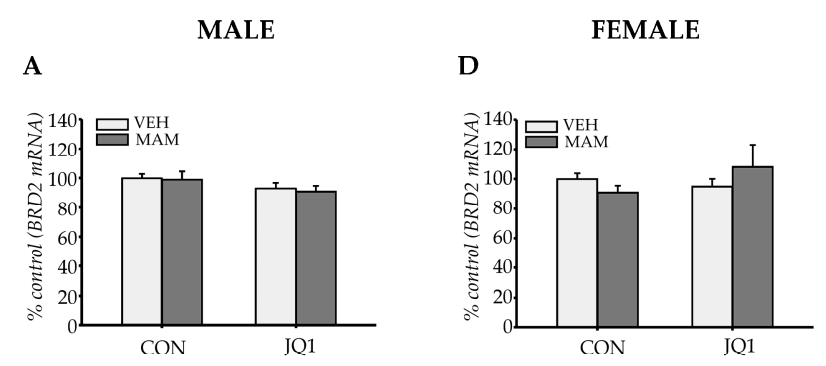

B

E
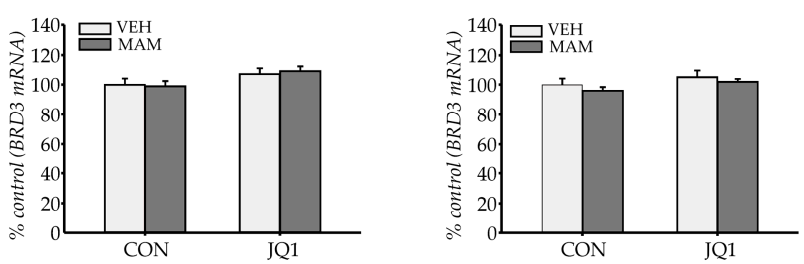

C

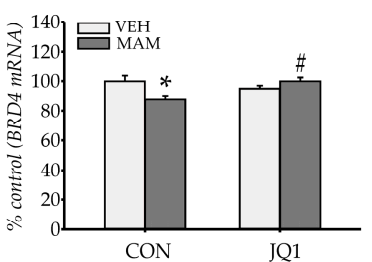

$\mathbf{F}$

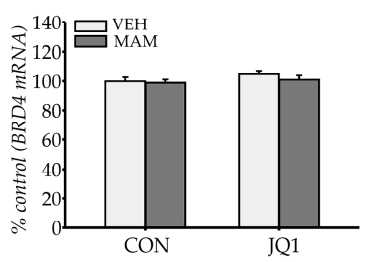

Figure 5. The effects of an adolescent JQ1 administration on $B R D$ gene expression in the adult medial prefrontal cortex in the MAM-E17 model of schizophrenia. The rats (males: A-C and females: D-F) were exposed to JQ1 or vehicle (CON) in early adolescence (P23-P29), and the analyses were performed in rats at P110. (A-C) BRD2, BRD3, and BRD4 expression in males, respectively. (D-F) $B R D 2, B R D 3$, and $B R D 4$ expression in females, respectively. The data are expressed as percentage of VEH-CON. Each data point represents the mean \pm SEM; $\mathrm{n}=6$ per group; ${ }^{*} p$ textless 0.05 vs. VEH-CON, \# $p$ textless 0.05 vs. MAM-CON (two-way ANOVA followed by a Tukey test).

The lack of an effect of MAM on BRD2 expression was also observed in the female mPFC (prenatal factor: $\mathrm{F}(1,20)=0.04 ; p=0.84$ ), and no interaction was observed between the two analysed factors (prenatal $\times$ postnatal factors: $\mathrm{F}(1,20)=1.28 ; p=0.27$, Figure 5D).

- $\quad$ BRD3 expression

Prenatal MAM administration did not alter the expression of the BRD3 mRNA in the mPFC of males (prenatal factor: $\mathrm{F}(1,20)=0.04 ; p=0.84$ ), but an effect of adolescent JQ1 treatment was observed (postnatal factor: $\mathrm{F}(1,20)=5.94 ; p$ textless 0.03 ). However, a statistically significant interaction between MAM and JQ1 treatment was not found (prenatal $\times$ postnatal factors: $\mathrm{F}(1,20)=0.17 ; p=0.69$, Figure $5 \mathrm{~B})$.

Prenatal MAM administration or an interaction between analysed factors did not alter $B R D 3$ expression in the female mPFC (prenatal factor: $\mathrm{F}(1,20)=1.53 ; p=0.23$ and prenatal $\times$ postnatal factors: $\mathrm{F}(1,20)=0.017 ; p=0.89$, Figure $5 \mathrm{E})$.

\section{- $\quad$ BRD4 expression}

Neither of the analysed factors affected BRD4 expression in the male mPFC (prenatal factor: $\mathrm{F}(1,20)=2.1 ; p=0.16$, postnatal factor: $\mathrm{F}(1,20)=1.64 ; p=0.21)$. However, a statistically significant interaction between factors was identified (prenatal $\times$ postnatal 
factors: $\mathrm{F}(1,20)=12.86 ; p$ textless 0.002$)$. A decrease in BRD4 mRNA levels was observed in the MAM-CON group ( $p$ textless 0.001 compared with the VEH-CON group), and this effect was abolished by adolescent JQ1 administration ( $p=0.99$ compared with the VEH-CON group and $p$ textless 0.02 compared with the MAM-CON group, Figure 5C).

In contrast, neither MAM nor JQ1 affected BRD4 mRNA levels in the female mPFC (prenatal factor: $\mathrm{F}(1,20)=1.22 ; p=0.28$, postnatal factor: $\mathrm{F}(1,20)=2.78 ; p=0.11)$. An interaction between analysed factors was not observed (prenatal $\times$ postnatal factors: $\mathrm{F}(1$, 20) $=0.4 ; p=0.53$, Figure 5F).

Thus, prenatal MAM administration decreased BRD4 expression only in males, and this effect was reversed by adolescent JQ1 treatment. However, neither MAM nor JQ1 administration affected $B R D 2$ and $B R D 3$ expression in the adult $\mathrm{mPFC}$ of either sex.

\subsubsection{Immediate Early Genes}

We analysed levels of the $c$-Fos, Npas4, and Arc mRNAs as markers of cellular activity in the mPFC of both males and females to determine whether adolescent JQ1 administration affected neuronal and synaptic activity at the molecular level.

- $\quad c$-Fos expression

Prenatal MAM administration did not affect $c$-Fos expression in the male mPFC (prenatal factor: $\mathrm{F}(1,20)=0.0014 ; p=0.97)$; however, an effect of JQ1 treatment was observed (postnatal factor: $\mathrm{F}(1,20)=0.57 ; p$ textless 0.0002$)$. An interaction between factors was not noted (prenatal $\times$ postnatal factors: $\mathrm{F}(1,20)=0.13 ; p=0.72)$. A decrease in $c-F o s$ mRNA levels was observed in the VEH-JQ1 and MAM-JQ1 groups ( $p$ textless 0.03 and $p$ textless 0.02 compared with the VEH-CON group respectively, Figure 6A).

In contrast, MAM altered $c$-Fos expression in the female $\mathrm{mPFC}$ (prenatal factor: $\mathrm{F}(1$, $20)=7.45 ; p$ textless 0.02$)$; however, JQ1 did not exert any effect (postnatal factor: F (1, $20)=0.01 ; p=0.91$ ). An interaction between analysed factors was not observed (prenatal $\times$ postnatal factors: $\mathrm{F}(1,20)=0.93 ; p=0.35)$, and a difference in mRNA levels between groups was not detected (Figure $6 \mathrm{D}$ ).

- $\quad$ Npas4 expression

MAM administration did not alter Npas4 expression in the male mPFC (prenatal factor: $\mathrm{F}(1,20)=0.02 ; p=0.88)$; however, JQ1 treatment exerted a significant effect (postnatal factor: $\mathrm{F}(1,20)=22.4 ; p$ textless 0.0002$)$. An interaction between factors was not observed (prenatal $\times$ postnatal factors: $\mathrm{F}(1,20)=0.29 ; p=0.59)$. A significant decrease in the level of the Npas4 mRNA was observed in the VEH-JQ1 and MAM-JQ1 groups ( $p$ textless 0.007 and $p$ textless 0.02 compared with the VEH-CON group respectively, Figure $6 \mathrm{~B}$ ).

MAM treatment also did not alter Npas4 expression in the female mPFC (prenatal factor: $\mathrm{F}(1,20)=0.24 ; p=0.63$ ), and JQ1 treatment exerted a significant effect (postnatal factor: $\mathrm{F}(1,20)=4.4 ; p$ textless 0.05$)$. Moreover, an interaction between factors was observed (prenatal $\times$ postnatal factors: $\mathrm{F}(1,20)=7.73 ; p$ textless 0.02$)$. A significant decrease in the level of the Npas4 mRNA was observed only in the VEH-JQ1 group ( $p$ textless 0.02 compared with the VEH-CON group, Figure 6E).

- Arc expression

MAM administration altered Arc expression in the male mPFC (prenatal factor: $\mathrm{F}$ $(1,20)=5.03 ; p$ textless 0.04$)$. However, JQ1 treatment did not modulate the level of the Arc mRNA (postnatal factor: F $(1,20)=3.22 ; p=0.088$ ), and no interaction was observed between the analysed factors (prenatal $\times$ postnatal factors: $F(1,20)=0.69$; $p=0.41$ ). A significant increase in the Arc mRNA level was detected in the MAM-JQ1 group ( $p$ textless 0.05 compared with the VEH-CON group, Figure $6 \mathrm{C}$ ).

Similar alterations were observed in females, and MAM treatment altered Arc expression in the female mPFC (prenatal factor: $\mathrm{F}(1,20)=13.37 ; p$ textless 0.002). JQ1 did not change the level of the Arc mRNA (postnatal factor: F $(1,20)=0.86 ; p=0.36$ ), and no interaction was observed between the analysed factors (prenatal $\times$ postnatal factors: 
$\mathrm{F}(1,20)=1.67 ; p=0.21)$. A significant increase in $\operatorname{Arc}$ mRNA levels was detected in the MAM-JQ1 group ( $p$ textless 0.02 compared with the VEH-CON group, Figure 6 F).
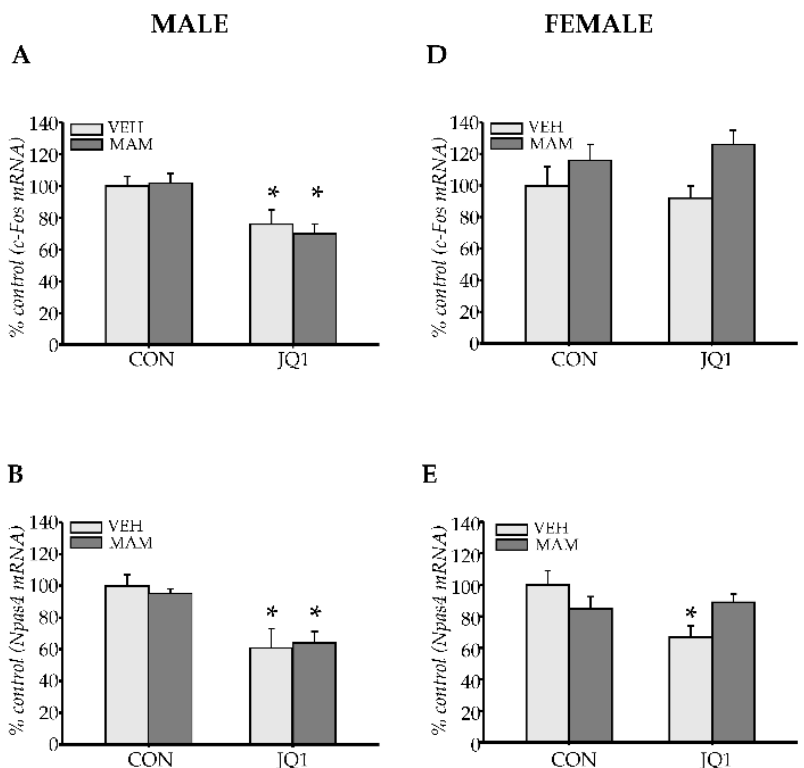

E
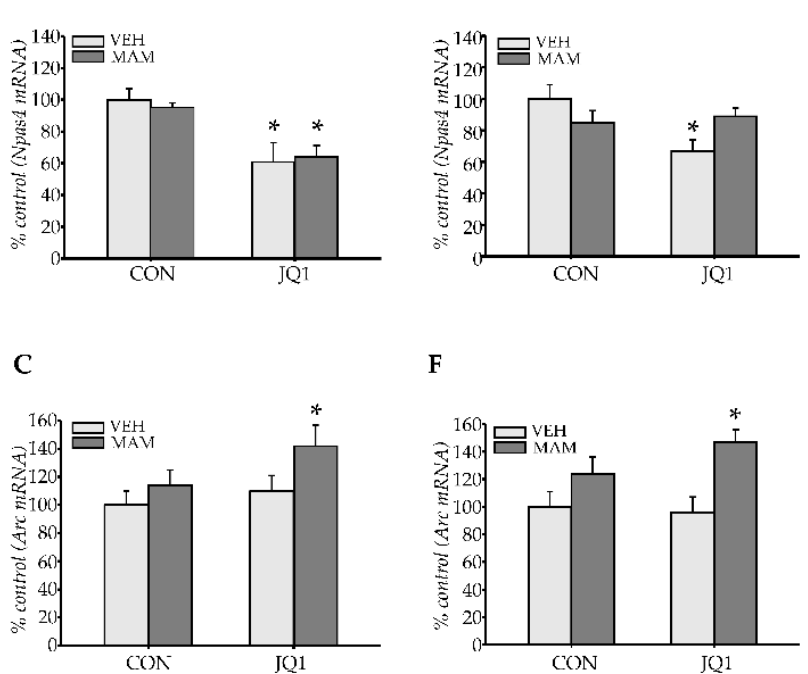

F

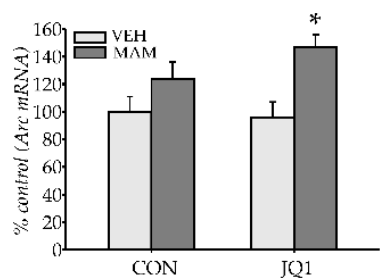

Figure 6. The effects of an adolescent JQ1 administration on early gene expression (c-Fos, Arc, Npas4) in the adult medial prefrontal cortex in the MAM-E17 model of schizophrenia. The rats (males: A-C and females: D-F) were exposed to JQ1 or vehicle (CON) in early adolescence (P23-P29), and the analyses were performed in rats at P110. (A-C) c-Fos, Arc, and Npas4 expression in males, respectively. (D-F) c-Fos, Arc, and Npas4 expression in females, respectively. The data are expressed as percentage of VEH-CON. Each data point represents the mean $\pm \mathrm{SEM} ; \mathrm{n}=6$ per group ${ }^{*} p$ textless 0.05 vs. VEH-CON (two-way ANOVA followed by a Tukey test).

Thus, prenatal MAM administration did not alter expression of the analysed immediate early genes in the adult mPFC of both sexes. However, JQ1 treatment repressed transcription of $c$-Fos and Npas4 genes in both the VEH and MAM groups of males, but in females, the Npas4 mRNA level was decreased only in the VEH group. In contrast, JQ1 administration increased Arc expression in the MAM-treated groups of both sexes.

\subsubsection{GABAergic Markers}

The expression of the GAD67 and $P V$ mRNAs was examined to determine whether adolescent inhibition of BET proteins affected GABAergic interneurons.

- GAD67 expression

MAM administration altered GAD67 expression in the male mPFC (prenatal factor: $\mathrm{F}$ $(1,20)=7.14 ; p$ textless 0.02$)$. However, JQ1 treatment did not change the level of the GAD67 mRNA (postnatal factor: $\mathrm{F}(1,20)=0.35 ; p=0.56$ ), and no interaction was observed between the analysed factors (prenatal $\times$ postnatal factors: $\mathrm{F}(1,20)=2.76 ; p=0.11)$. A significant decrease in GAD67 mRNA levels was detected in the MAM-CON group ( $p$ textless 0.03 compared with the VEH-CON group, Figure 7A). 
MALE

A

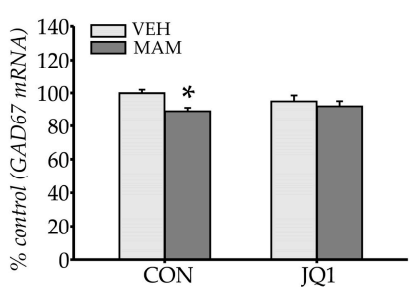

C

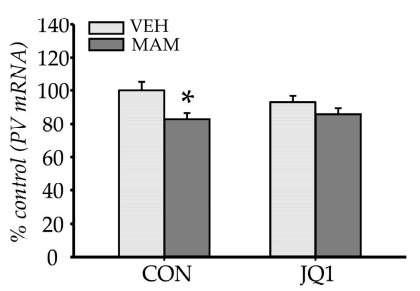

FEMALE

B

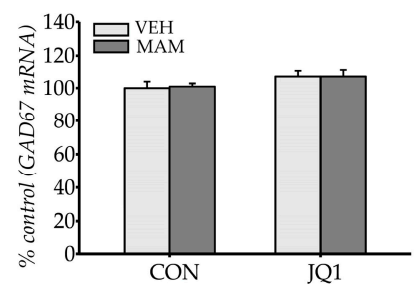

D

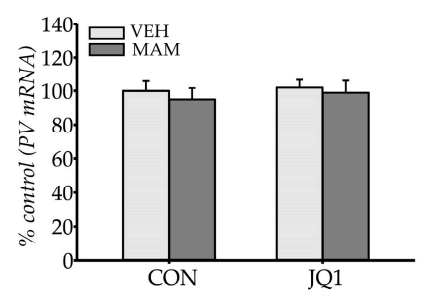

Figure 7. The effects of an adolescent JQ1 administration on GAD67 and parvalbumin (PV) gene expression in the adult medial prefrontal cortex in the MAM-E17 model of schizophrenia. The rats (males and females) were exposed to JQ1 or vehicle (CON) in early adolescence (P23-P29), and the analyses were performed in rats at P110. (A,C) GAD67 and $P V$ expression in males, respectively. (B,D) GAD67 and $P V$ expression in females, respectively. The data are expressed as percentage of VEH-CON. Each data point represents the mean \pm SEM; $\mathrm{n}=6$ per group ${ }^{*} p$ textless 0.05 vs. VEH-CON (two-way ANOVA followed by a Tukey test).

Prenatal MAM administration did not alter GAD67 expression in the female mPFC (prenatal factor: $\mathrm{F}(1,20)=0.016 ; p=0.90)$; however, an effect of JQ1 treatment was observed (postnatal factor: $\mathrm{F}(1,20)=4.55 ; p$ textless 0.05$)$. An interaction between factors was not noted (prenatal $\times$ postnatal factors: $\mathrm{F}(1,20)=0.072 ; p=0.79)$, and no statistically significant differences in GAD67 mRNA levels were observed among groups (Figure 7B).

- $\quad P V$ expression

MAM administration altered $P V$ expression in the male mPFC (prenatal factor: $\mathrm{F}$ $(1,20)=10.51 ; p$ textless 0.005$)$. However, JQ1 treatment did not change the level of the $P V$ mRNA (postnatal factor: $\mathrm{F}(1,20)=0.33 ; p=0.57$ ), and no interaction was observed between the analysed factors (prenatal $\times$ postnatal factors: $\mathrm{F}(1,20)=1.86 ; p=0.19$ ). A significant decrease in $P V$ mRNA levels was detected in the MAM-CON group ( $p$ textless 0.02 compared with the VEH-CON group, Figure 7C).

Neither of the analysed factors affected $P V$ expression in the female mPFC (prenatal factor: $F(1,20)=0.45 ; p=0.51$, postnatal factors: $F(1,20)=0.26 ; p=0.62)$. An interaction between the analysed factors was not observed (prenatal $\times$ postnatal factors: $F(1$, 20) $=0.052 ; p=0.82$, Figure 7D).

Thus, the prenatal MAM factor decreased both GAD67 and $P V$ expression only in the male $\mathrm{mPFC}$ of the MAM-treated group. Postnatal JQ1 administration did not affect either GAD67 or $P V$ expression in either sex. 


\subsubsection{Correlation Analysis of Gene Expression and Behavioural Response}

The behavioural tests and gene expression analyses were completed on the same animals. Thus, a correlation analysis was performed to determine the associations between behavioural responses and changes in gene expression in the adult mPFC (Figure 8).
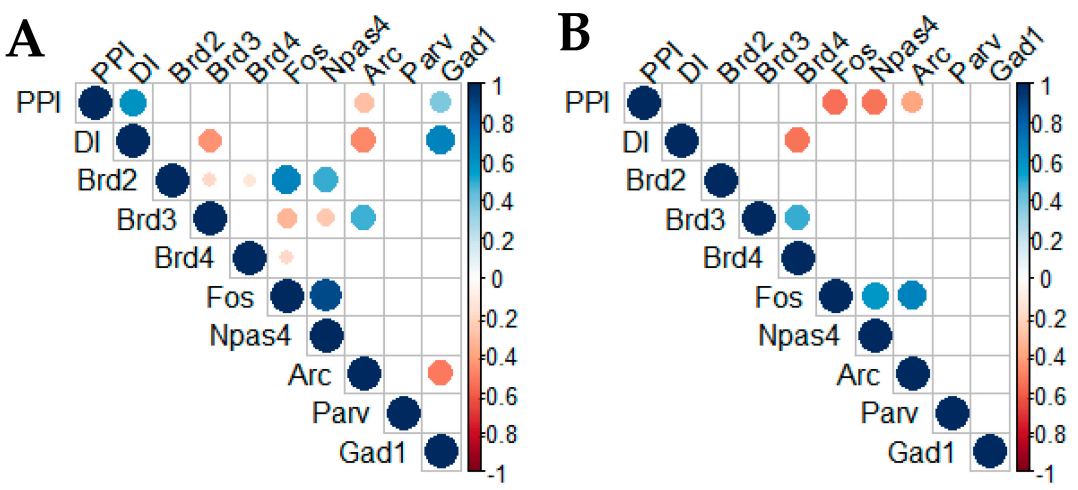

Figure 8. The matrix of correlation plots demonstrates correlations among the levels of the studied mRNA's in the adult medial prefrontal cortex and behavioural data in males (A) and females (B) (Pearson's correlation, $p$ textless 0.05). PPI-pre-pulse inhibition, DI-discrimination index. The colour code follows the indicated values of the correlation coefficient. The colour intensity and the size of the circles are proportional to the correlation coefficients. Two basic colours represent negative (red) or positive (blue) correlations.

The correlation matrix constructed for sensorimotor gating results (PPIs) in males revealed a positive correlation with object recognition data (DI) (Figure 8A). This effect was not observed in females (Figure 8B).

In males, PPI and DI showed positive correlations with the expression of the GAD67 mRNA (Gad1 gene), but they were negatively correlated with Arc expression. In addition, the expression of the $B R D 3 \mathrm{mRNA}$ exhibited a negative correlation with the results of the novel object recognition test. The level of the BRD2 mRNA was proportional to the expression of the $c$-Fos and Npas4 mRNAs, while BRD3 expression was inversely proportional to the levels of these mRNAs. In contrast, $B R D 3$ expression was positively correlated with Arc expression. Moreover, c-Fos expression was positively correlated with Npas4 expression, while Arc levels exhibited a negative correlation with the GAD67 mRNA (Figure 8A). Thus, deficits in recognition memory and sensorimotor gating in males appear to be related to GAD67 and Arc expression. Moreover, recognition memory formation appears to be correlated with BRD3 and immediate early genes' (c-Fos, Npas4, Arc) transcription activity.

In females, PPI negatively correlated with immediate early gene expression (c-Fos, Npas4, and $A r c$ ), and DI was negatively correlated with the level of the BRD4 mRNA. However, $B R D 4$ expression exhibited a positive correlation with the BRD3 mRNA level. In addition, c-Fos expression was positively correlated with Npas4 and Arc gene transcription (Figure 8B). Thus, a deficit in sensorimotor gating in females appears to be related to immediate early gene expression (c-Fos, Npas4, and Arc), while recognition memory formation is rather correlated with transcription of $B R D$ genes (BRD4, BRD3).

\subsection{Proteomic Analysis of the Adult Medial Prefrontal Cortex}

A proteomic study was performed to determine whether adolescent BET protein inhibition altered the protein landscape in the adult mPFC.

\subsubsection{Proteomic Analysis of Protein Expression in Males}

iTRAQ identified 313 proteins and 1049 unique peptides. Among them, 48 proteins were identified as differentially expressed (Table 1). 
Table 1. The list of 48 proteins identified in the adult medial prefrontal cortex of the male rats in the proteomic analysis. For each protein match, Mascot calculates an overall Protein Score. This number reflects the combined scores of all observed mass spectra that can be matched to amino acid sequences within that protein. A higher score indicates a more confident match.

\begin{tabular}{|c|c|c|c|c|c|}
\hline Accession & Gene & Protein & MW.kDa & Scores & Peptides \\
\hline ADT1 & Slc25a4 & ADP / ATP translocase 1 & 33 & 139.5 & 5 \\
\hline ADT2 & Slc25a5 & ADP / ATP translocase 2 & 32.9 & 96.9 & 4 \\
\hline AP2A2 & Ap2a2 & AP-2 complex subunit alpha-2 & 104 & 293.2 & 4 \\
\hline AP2B1 & Ap2b1 & AP-2 complex subunit beta & 104.5 & 109.5 & 3 \\
\hline ARP3 & Actr3 & Actin-related protein 3 & 47.3 & 46.6 & 1 \\
\hline AT1A2 & Atp1a2 & $\begin{array}{l}\text { Sodium/potassium-transporting ATPase } \\
\text { subunit alpha-2 }\end{array}$ & 112.1 & 220.5 & 3 \\
\hline ATPB & Atp5f1b & ATP synthase subunit beta, mitochondrial & 56.3 & 748.1 & 12 \\
\hline ATPD & Atp5d & ATP synthase subunit delta & 17.6 & 54.6 & 2 \\
\hline CALM & Calm1 & Calmodulin & 16.8 & 98.8 & 2 \\
\hline CAZA2 & Capza2 & F-actin-capping protein subunit alpha-2 & 32.9 & 68.5 & 1 \\
\hline CISY & Cs & Citrate synthase & 23.9 & 59.6 & 1 \\
\hline CLH1 & Cltc & Clathrin heavy chain 1 & 191.4 & 671 & 13 \\
\hline CNTN1 & Cntn1 & Contactin-1 & 113.4 & 158.2 & 4 \\
\hline CPLX1 & Cplx1 & Complexin-1 & 15.1 & 41.4 & 1 \\
\hline CTNB1 & Ctnnb1 & Catenin beta- 1 & 85.4 & 75.3 & 1 \\
\hline DCLK1 & Dclk1 & Serine/threonine-protein kinase DCLK1 & 84.1 & 51.8 & 1 \\
\hline DPYL2 & Dpysl2 & Dihydropyrimidinase-related protein 2 & 62.2 & 618.8 & 8 \\
\hline DPYL4 & Dpysl4 & Dihydropyrimidinase-related protein 4 & 61 & 62.6 & 2 \\
\hline ENOA & Eno1 & Alpha-enolase & 23.8 & 123.7 & 2 \\
\hline GABT & Abat & 4-aminobutyrate aminotransferase & 56.4 & 80.5 & 2 \\
\hline GDIA & Gdi1 & Rab GDP dissociation inhibitor alpha & 50.5 & 164 & 3 \\
\hline GLNA & Glul & Glutamine synthetase & 42.1 & 148.5 & 2 \\
\hline GMFB & $\mathrm{Gmfb}$ & Glia maturation factor beta & 16.7 & 73.1 & 1 \\
\hline GNAO & Gnao1 & $\begin{array}{c}\text { Guanine nucleotide-binding protein } \mathrm{G}(\mathrm{o}) \\
\text { subunit alpha }\end{array}$ & 40 & 198 & 3 \\
\hline KAD1 & Ak1 & Adenylate kinase isoenzyme 1 & 21.6 & 75.5 & 1 \\
\hline KAPCB & Prkacb & $\begin{array}{l}\text { cAMP-dependent protein kinase catalytic } \\
\text { subunit beta }\end{array}$ & 40.7 & 39.5 & 1 \\
\hline KCRU & Ckmt1 & Creatine kinase U-type, mitochondrial & 47 & 112.7 & 2 \\
\hline KPCG & Prkcg & Protein kinase $C$ gamma & 78.3 & 40.8 & 1 \\
\hline KPYM & $\mathrm{Pkm}$ & Pyruvate kinase PKM & 57.8 & 440.6 & 9 \\
\hline $\mathrm{MDHC}$ & Mdh1 & Malate dehydrogenase & 36.5 & 48.5 & 2 \\
\hline MTAP2 & Map2 & Microtubule-associated protein 2 & 199 & 112.8 & 1 \\
\hline MYPR & Plp1 & Myelin proteolipid protein & 30.1 & 54.7 & 1 \\
\hline NCALD & Ncald & Neurocalcin-delta & 22.2 & 46.4 & 1 \\
\hline NCAM1 & Ncam1 & Neural cell adhesion molecule 1 & 94.6 & 79.6 & 2 \\
\hline NDUBA & Ndufb10 & $\begin{array}{l}\text { NADH dehydrogenase [ubiquinone] } 1 \text { beta } \\
\text { subcomplex subunit } 10\end{array}$ & 21 & 45.9 & 1 \\
\hline NEUM & Gap43 & Neuromodulin & 23.6 & 132.3 & 4 \\
\hline NSF & Nsf & Vesicle-fusing ATPase & 82.5 & 144.4 & 5 \\
\hline PGK1 & Pgk1 & Phosphoglycerate kinase 1 & 44.5 & 187 & 4 \\
\hline RAP1B & Rap1b & Ras-related protein Rap- $1 b$ & 20.8 & 73.2 & 1 \\
\hline SDHA & Sdha & $\begin{array}{l}\text { Succinate dehydrogenase [ubiquinone] } \\
\text { flavoprotein subunit, mitochondrial }\end{array}$ & 60 & 68.5 & 1 \\
\hline SUCA & Suclg1 & $\begin{array}{l}\text { Succinyl-CoA ligase [ADP/GDP-forming] } \\
\text { subunit alpha, mitochondrial }\end{array}$ & 36.1 & 74.3 & 1 \\
\hline SYN2 & Syn2 & Synapsin-2 & 63.3 & 114.9 & 3 \\
\hline SYUB & Sncb & Beta-synuclein & 14 & 47.4 & 1 \\
\hline TENR & Tnr & Tenascin- $\mathrm{R}$ & 149.5 & 124.8 & 2 \\
\hline VATA & Atp6v1a & V-type proton ATPase catalytic subunit A & 68.3 & 357.9 & 4 \\
\hline VATL & Atp6v0c & $\begin{array}{c}\text { V-type proton ATPase } 16 \text { kDa proteolipid } \\
\text { subunit }\end{array}$ & 15.8 & 40.9 & 1 \\
\hline VDAC1 & Vdac1 & $\begin{array}{l}\text { Voltage-dependent anion-selective channel } \\
\text { protein } 1\end{array}$ & 32.3 & 504.4 & 7 \\
\hline VGLU1 & Slc17a7 & Vesicular glutamate transporter 1 & 61.6 & 127.5 & 2 \\
\hline
\end{tabular}


The lowest Mascot score of the identified proteins was 35, with molecular masses ranging from 2 to $532 \mathrm{kDa}$ (median $48 \mathrm{kDa}$ ). The heatmap in Figure 9A shows the protein expression levels in the analysed groups and a functional clustering analysis of the identified proteins. Proteins with similar expression patterns have relatively shorter Euclidean distances. Hierarchical clustering analysis with the Euclidean distance of proteins was used to identify patterns of protein expression following prenatal and postnatal treatment. Proteins were classified into 4 categories: 3 proteins (NEUM, SYUB, and ATPD) were affected by MAM treatment and their levels decreased mainly in the MAM-CON group (I); 26 proteins (CNTN1, MDHC, CALM, CTNB1, SUCA, CAZA2, AP2B1, AT1A2, MYPR, MTAP2, NCAM1, GLNA, CPLX1, KPCG, TENR, GDIA, ADT1, ADT2, PGK1, KPYM, SYN2, NSF, VGLU1, DPYL4, GNAO, and VDAC1) were altered by JQ1 treatment and their levels were increased in the VEH-JQ1 and MAM-JQ1 groups (II); 10 proteins (DPYL2, GMFB, DCLK1, NCALD, ENOA, RAP1B, ARP3, KAD1, ATPB, and KCRU) were also modulated by JQ1 treatment and their levels were decreased in the VEH-JQ1 and MAM-JQ1 groups (III); 9 proteins (VATL, AP2A2, VATA, NDUBA, CISY, CLH1, SDHA, GABT, and KAPCB) were altered by MAM and their levels were mainly increased in the MAM-CON group (IV).

- Principal component analysis (PCA)

PCA was conducted to identify unique components related to the interaction between both treatments: prenatal (MAM) and adolescent (JQ1). The first three components (Dim1, Dim2, Dim3) defined 70\% of the total variance in our experiment (Figure 9B). An examination of the scatter plots of the proteins' principal components revealed that the first component was linked to clusters II and III from the previous classification, namely, proteins regulated by adolescent JQ1 treatment (Figure 9B). The second component, reflecting reciprocally affected clusters I and IV from the previous analysis, identified proteins affected by prenatal MAM administration (Figure 9B). The third component corresponded to proteins classified in different defined clusters from a previous analysis; however, it comprised proteins functionally related to synapse and cytoskeleton organisation (AP2B1, CAZA2, DPYL2, MYPR, NEUM, SYUB, and VGLU1) or energy transfer processes (AT1A2, ATPB, KCRU, and VATL) (Figure 9B).

The two first components (Dim1, Dim2) differentiate all experimental groups (Figure 9C).

The proteomic analysis in the male adult mPFC identified distinctive molecular signatures related to either prenatal factor (MAM administration) or postnatal factor (adolescent JQ1 treatment) action.

2.4.2. Proteomic Analysis of Protein Expression in Females

iTRAQ identified 348 proteins and 1190 unique peptides. Among them, 26 proteins were identified as differentially expressed (Table 2). 

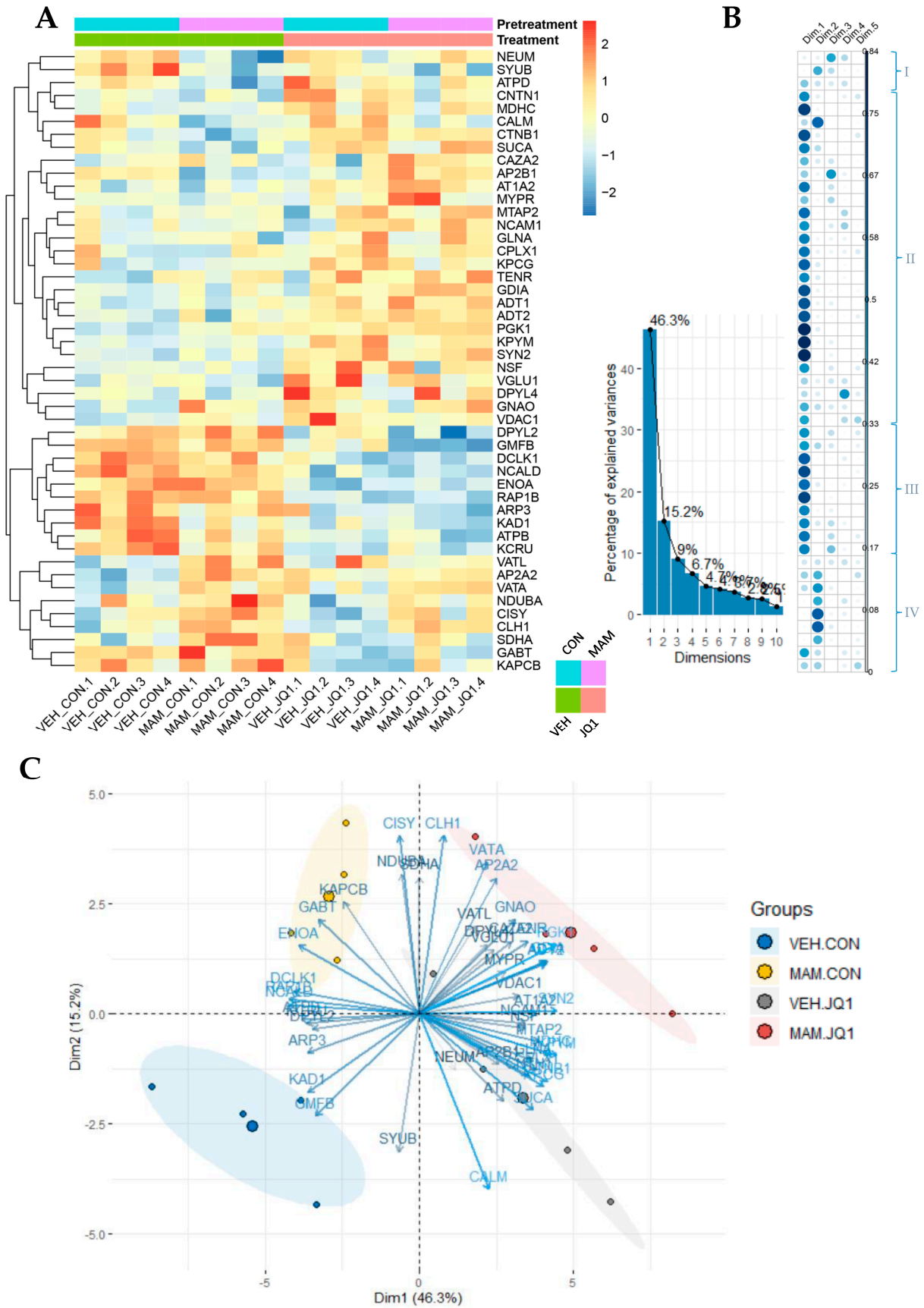

Figure 9. Proteomics study in the adult medial prefrontal cortex of males in the MAM-E17 model of schizophrenia. The rats were exposed to JQ1 or vehicle (CON) in early adolescence (P23-P29) and the analyses were performed in rats at P110. (A) Heatmap view of 48 differentially expressed proteins across groups ( $p$ textless 0.050 ) identified from iTRAQ analysis. The colour scale illustrates the relative expression level of each protein across the 4 samples (biological replicates of mPFC) of each group: VEH-CON (1-4), MAM-CON (1-4), VEH-JQ1 (1-4), and MAM-JQ1 (1-4). Red and blue indicate higher and lower expression than the median expression value, respectively. The colour palette (right bar) represents standardised protein expression values (from red: upregulated, to blue: downregulated proteins). (B) Correlogram of the representation of the differentially expressed proteins depicted in the heatmap as well as their contribution to the principal components (Dim1-Dim5). Protein clusters (I-IV) on the right correspond to proteins visualised on the heatmap and correlogram. (C) PC biplot of the differentially expressed proteins. On the whole, the first component is linked to clusters affected by adolescent JQ1 treatment (cluster II and III). The second component corresponds to clusters affected by prenatal MAM administration (cluster I and IV). The first two principal components enabled us to visualise proteins specific for each treatment as well as the interaction between MAM and JQ1 administration. Abbreviated protein names are defined in Table 1. 
Table 2. The list of 26 proteins identified in the adult medial prefrontal cortex of the female rats in the proteomic analysis. For each protein match, Mascot calculates an overall Protein Score. This number reflects the combined scores of all observed mass spectra that can be matched to amino acid sequences within that protein. A higher score indicates a more confident match.

\begin{tabular}{|c|c|c|c|c|c|}
\hline Accession & Gene & Protein & MW.kDa & Scores & Peptides \\
\hline AT1A1 & Atp1a1 & $\begin{array}{c}\text { Sodium/potassium-transporting ATPase } \\
\text { subunit alpha-1 }\end{array}$ & 112.9 & 439.5 & 6 \\
\hline CAND1 & Cand1 & Cullin-associated NEDD8-dissociated protein 1 & 136.2 & 176 & 3 \\
\hline CLH1 & Cltc & Clathrin heavy chain 1 & 191.4 & 703.3 & 12 \\
\hline CNTN1 & Cntn1 & Contactin-1 & 113.4 & 88.6 & 2 \\
\hline $\mathrm{COX} 5 \mathrm{~B}$ & Cox $5 b$ & Cytochrome $\mathrm{c}$ oxidase subunit $5 \mathrm{~B}$, mitochondrial & 13.9 & 51.7 & 2 \\
\hline DCLK1 & Dclk1 & Serine/threonine-protein kinase DCLK1 & 84.1 & 61.3 & 1 \\
\hline DPYL4 & Dpysl4 & Dihydropyrimidinase-related protein 4 & 61 & 111.7 & 2 \\
\hline EAA1 & Slc1a3 & Excitatory amino acid transporter 1 & 59.6 & 69 & 1 \\
\hline EF1A2 & Eef1a2 & Elongation factor 1-alpha 2 & 50.4 & 153.5 & 4 \\
\hline EFTU & Tufm & Elongation factor $\mathrm{Tu}$, mitochondrial & 18.8 & 54.4 & 1 \\
\hline ENOG & Eno2 & Gamma-enolase & 47.1 & 263 & 5 \\
\hline FKB1A & Fkbp1a & Peptidyl-prolyl cis-trans isomerase FKBP1A & 11.9 & 38.8 & 1 \\
\hline HINT1 & Hint1 & Histidine triad nucleotide-binding protein 1 & 13.8 & 79.1 & 1 \\
\hline KPYM & Pkm & Pyruvate kinase PKM & 57.8 & 467.5 & 10 \\
\hline LDHC & Ldhc & L-lactate dehydrogenase $\mathrm{C}$ chain & 35.7 & 53 & 1 \\
\hline NDUV2 & Ndufv2 & $\begin{array}{l}\text { NADH dehydrogenase [ubiquinone] } \\
\text { flavoprotein } 2, \text { mitochondria }\end{array}$ & 27.4 & 69.4 & 1 \\
\hline PGK1 & Pgk1 & Phosphoglycerate kinase 1 & 44.5 & 213.2 & 4 \\
\hline SPTN1 & Sptan1 & Spectrin alpha chain, non-erythrocytic 1 & 284.5 & 951.5 & 18 \\
\hline SPTN2 & Sptbn2 & Spectrin beta chain, non-erythrocytic 2 & 270.9 & 100.4 & 2 \\
\hline SYN1 & Syn1 & Synapsin-1 & 73.9 & 284.5 & 6 \\
\hline SYUB & Sncb & Beta-synuclein & 14 & 65.4 & 2 \\
\hline TBB4A & Tubb4a & Tubulin beta- $4 \mathrm{~A}$ chain & 49.6 & 930.5 & 20 \\
\hline ТCPB & Cct2 & T-complex protein 1 subunit beta (Fragments) & 15.9 & 70.5 & 1 \\
\hline TENR & Tnr & Tenascin- $\mathrm{R}$ & 149.5 & 137.3 & 2 \\
\hline VATG2 & Atp6v1g2 & V-type proton ATPase subunit G 2 & 13.6 & 36.6 & 1 \\
\hline VDAC1 & Vdac1 & $\begin{array}{l}\text { Voltage-dependent anion-selective channel } \\
\text { protein } 1\end{array}$ & 30.7 & 546.4 & 7 \\
\hline
\end{tabular}

The lowest Mascot score of the identified proteins was 26, with molecular masses ranging from 2 to $532 \mathrm{kDa}$ (median $48 \mathrm{kDa}$ ). The heatmap in Figure 10A shows the protein expression levels in the analysed groups and a functional clustering analysis of the identified proteins. Proteins with similar expression patterns have relatively shorter Euclidean distances. Hierarchical clustering analysis with the Euclidean distance of proteins was used to identify patterns of protein expression following prenatal and postnatal treatment. Proteins were classified into 2 categories based on the effects of the JQ1 treatment: the levels of 9 proteins (AT1A1, NDUV2, TENR, EAA1, TCPB, CAND1, TBB4A, CLH1, and DCLK1) decreased in both the VEH-JQ1 and MAM-JQ1 groups (I), and the levels of 17 proteins (FKB1A, SPTN1, CNTN1, LDHC, ENOG, SYN1, DPYL4, SYUB, VATG2, COX5B, HINT1, EF1A2, PGK1, VDAC1, EFTU, KPYM, and SPTN2) increased in the VEH-JQ1 and MAM-JQ1 groups (II). 
A B

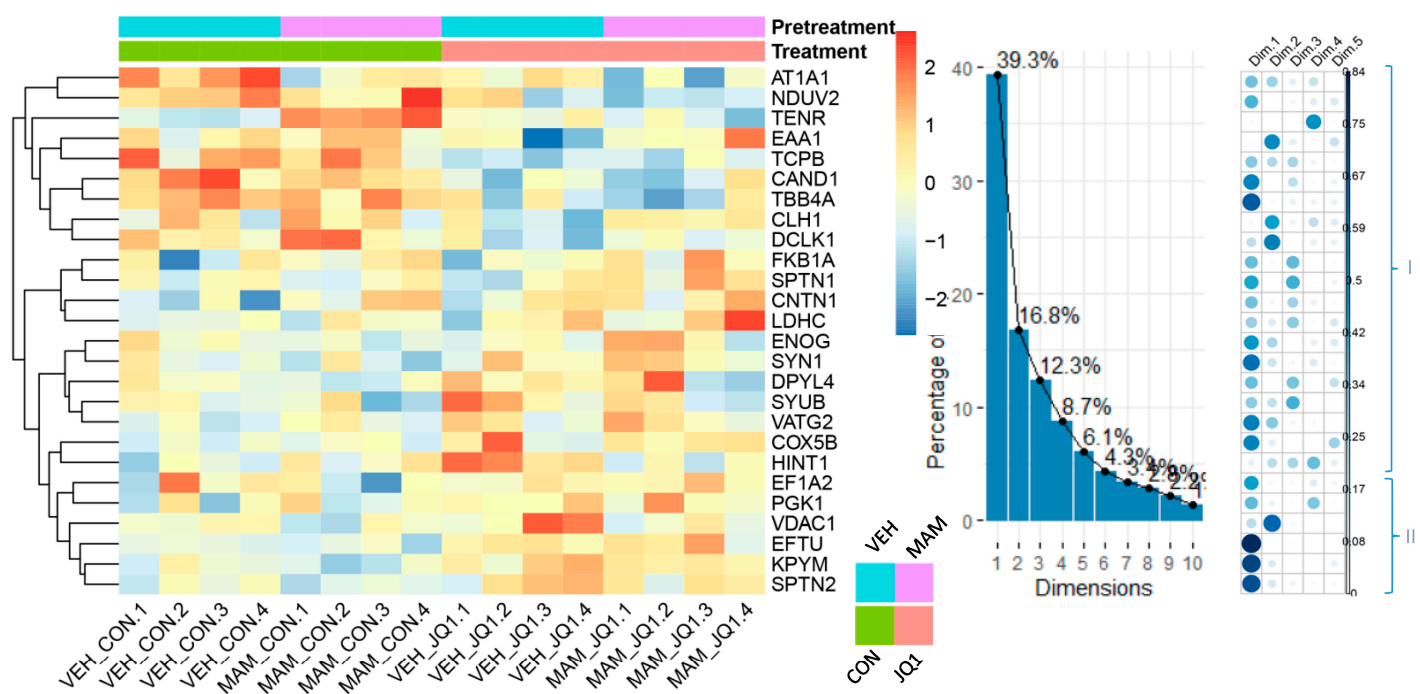

C

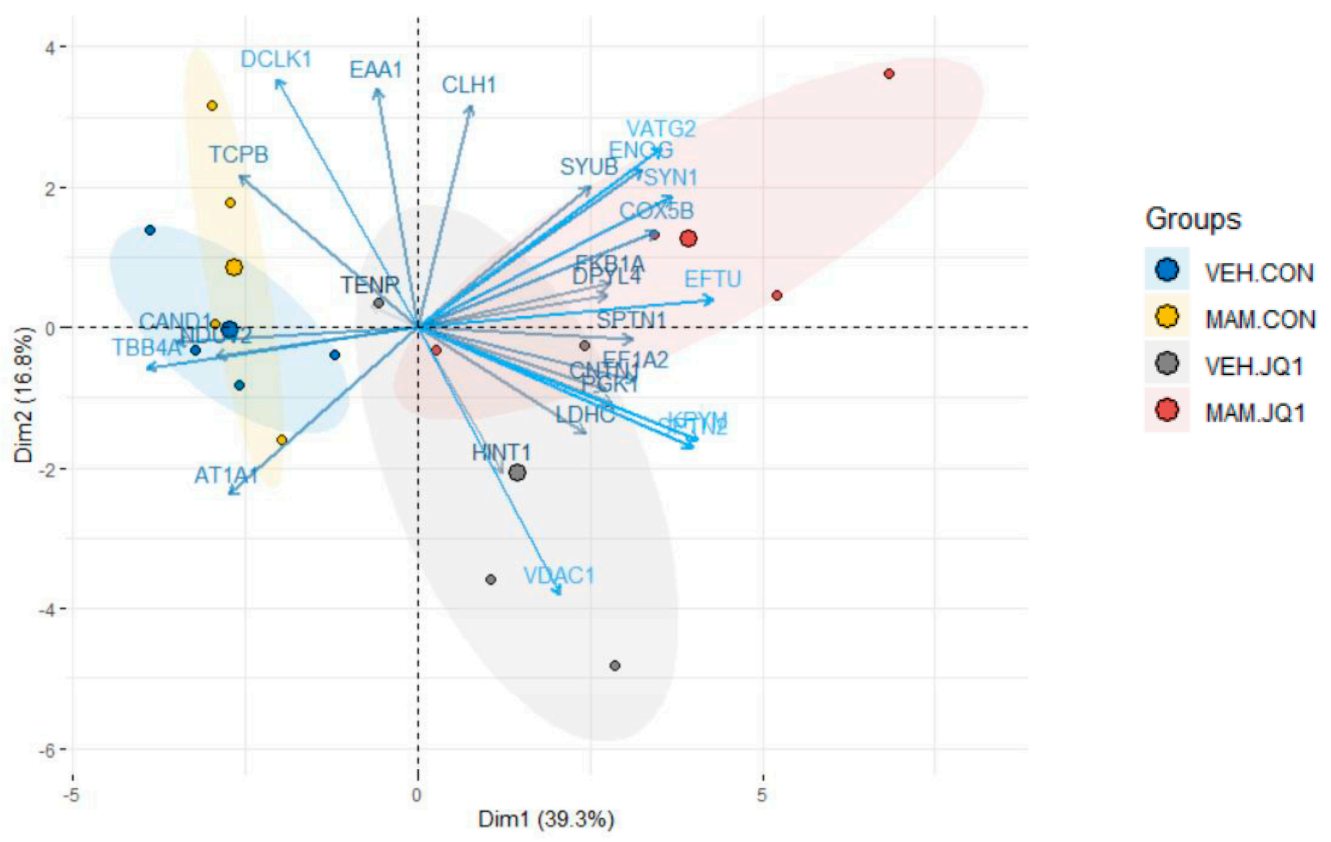

Figure 10. Proteomics study in the adult medial prefrontal cortex of females in the MAM-E17 model of schizophrenia. The rats were exposed to JQ1 or vehicle (CON) in early adolescence (P23-P29), and the analyses were performed in rats at P110. (A) Heatmap view of 26 differentially expressed proteins across groups ( $p$ textless 0.050 ) identified from iTRAQ analysis. The colour scale illustrates the relative expression level of each protein across the 4 samples (biological replicates of mPFC) of each group: VEH-CON (1-4), MAM-CON (1-4), VEH-JQ1 (1-4), and MAM-JQ1 (1-4). Red and blue indicate higher and lower expression than the median expression value, respectively. The colour palette (right bar) represents standardised protein expression values (from red: upregulated, to blue: downregulated proteins). (B) Correlogram of the representation of the differentially expressed proteins depicted in the heatmap as well as their contribution to the principal components (Dim1-Dim5). Protein clusters (I-II) on the right correspond to proteins visualised on the heatmap and correlogram. (C) PC biplot of the differentially expressed proteins. On the whole, both components are linked to clusters (I and II) affected by adolescent JQ1 treatment. The first two principal components enabled us to visualise proteins engaged in JQ1 treatment. Abbreviated protein names are defined in Table 2. 


\section{- $\quad$ Principal component analysis (PCA)}

PCA was used to identify unique components related to the interaction between both treatments: prenatal MAM and adolescent JQ1. The first three components (Dim1, Dim2, Dim3) defined $70 \%$ of the total variance in our experiment (Figure 10B). An examination of the scatter plots of the proteins' principal components revealed that the first component (Dim1) was linked to clusters I and II from the previous classification, indicating proteins regulated by adolescent JQ1 treatment (Figure 10B). Similar to Dim1, the second component, reflecting reciprocally affected clusters I and II (synaptic proteins: CLH1, DCLK1, and EAA1) from the previous analysis, identified proteins that were altered by adolescent JQ1 administration (Figure 10B). The third component corresponded to proteins classified in cluster I in the previous analysis (Figure 10B). The two first components (Dim1, Dim2) separate JQ1 groups from CON groups (Figure 10C).

Thus, proteomic analysis showed that changes in protein level in the female adult mPFC were related only to postnatal factor (adolescent JQ1 treatment).

\section{Discussion}

As shown in the present study, the administration of JQ1, an inhibitor of BET family proteins, in a defined period of adolescence affected behavioural responses and molecular and proteomic landscapes in the adult mPFC. The effects of JQ1 action were observed in animals of both sexes prenatally treated with VEH and MAM.

\subsection{Behavioural Response}

Behavioural tests were performed to analyse deficits in sensorimotor gating and recognition memory that are commonly observed in the MAM-E17 rat model of schizophrenia [30-33]. These behavioural impairments are considered models of the cognitive symptoms of schizophrenia. Sensorimotor gating is a pre-attentive process that is measured by PPI, and abnormalities in pre-attentive information processing may predict or lead to complex cognitive deficits [19]. PPI deficits were originally identified in patients with schizophrenia, and in preclinical studies, PPI was also used to investigate sensorimotor gating deficits in animal models of schizophrenia $[19,20]$. The memory impairment was examined using the novel object recognition test, which is known to have translational relevance to the domain of visual learning and memory [21,22], among the cognitive deficits observed in patients with schizophrenia [34]. The aforementioned task is a useful tool to evaluate recognition memory in animal models; however, some limitations related to the preference of a novel object should be considered, and the mechanisms involved in visual recognition memory of humans and rodents are not identical [21].

In the present study, we observed impairments in sensorimotor gating and memory recognition in male but not female MAM-E17 rats. Our previous studies also showed a decrease in PPI and memory impairments in the novel object recognition test in MAM-treated male rats $[17,18,35]$. However, in these studies, we did not analyse female rats. Literature data indicate some deficits in sensorimotor gating [36] or a reduction in discrimination index in novel object recognition in MAM-E17 females [29]. However, these studies were performed in Sprague-Dawley rats rather than in the Wistar Han rats used in our study. On the other hand, some clinical studies reported that healthy women showed lower levels of PPI than men [37,38], and a similar effect was reported in our study, where the PPI of males was higher than that of females. Moreover, the results from patients with schizophrenia are inconsistent, showing a decrease or unaffected sensorimotor gating in females [37,39], similar to those noted in the MAM-E17 model. Thus, female rats might not display all of the schizophrenia-like behavioural deficits induced by prenatal MAM treatment that were observed in male rats.

Adolescent JQ1 treatment affected the analysed behavioural parameters. In males, JQ1 impaired either memory recognition or sensorimotor gating in control rats (VEH-JQ1 group) but did not alter these parameters in the MAM group. In females, adolescent BET protein inhibition induced a reduction in novel object recognition in control rats; however, 
prenatal MAM treatment prevented this effect. On the other hand, JQ1 did not alter PPI but increased the startle amplitude in the VEH- and MAM-treated groups of female rats. To the best of our knowledge, the effect of JQ1 treatment has not yet been studied in acoustic startle response tests; thus, we are unable to easily compare our data with others. However, the role of BET proteins was determined in a novel object recognition test, and JQ1-treated adult animals exhibited impaired memory formation [12] and the blockade of an enhancement of recognition of a new object induced by an HDAC3 inhibitor [40]. Thus, our results add new knowledge of the role of BET proteins in the development of some behavioural responses, such as sensorimotor gating and recognition memory. Moreover, our data imply that the inhibition of BET family function during adolescence might disrupt these processes in adulthood, especially in males.

\subsection{LTP in the Adult mPFC}

The prefrontal cortex functionally and structurally transforms during the transition from adolescence to adulthood, which makes it very vulnerable to any environmental changes. Abnormal prefrontal cortical development might affect cognitive function in adulthood [41]. Thus, we analysed LTP formation in the adult MPFC to examine cortical cellular function, and we observed deficits in LTP only in the MAM-E17 male but not in the female $\mathrm{mPFC}$. Our results are consistent with previous findings [27], showing a decrease in LTP only in the mPFC of MAM-treated adult male, not female mice. Thus, the available data suggest that male mPFC development might be more vulnerable to prenatal MAM treatment than female $\mathrm{mPFC}$ development.

Adolescent JQ1 administration only exerted a slight insignificant effect on LTP formation in the mPFC of control adult animals of both sexes and did not affect the MAM groups. On the other hand, JQ1 might significantly increase LTP in mouse hippocampal slices [42]. Thus, the role of the BET protein in LTP formation might depend on the brain region and neurodevelopmental period, and our results did not indicate sex differences in the effect of JQ1 on LTP in the MPFC.

\subsection{Molecular Changes in the Adult mPFC}

We examined the effect of adolescent functional blockade of BET proteins on $B R D$ gene expression in the adult $\mathrm{mPFC}$. Prenatal MAM administration decreased BRD4 expression only in male rats, and it had no effect on BRD2 and BRD3 mRNA levels in either sex. To the best of our knowledge, this report is the first to show changes in $B R D$ expression in the $\mathrm{MPFC}$, which might suggest the involvement of the BET family in prefrontal cortical maturation and the development of schizophrenia-like abnormalities in male MAM-E17 rats. Adolescent JQ1 treatment did not affect $B R D$ expression; however, it abolished the MAM-induced decrease in BRD4 mRNA levels in the male mPFC. Thus, JQ1 administration during adolescence might normalise BRD cortical dysfunction.

In the present study, levels of IEGs (c-Fos, Arc, and Npas4) were examined as indirect markers of neuronal activity related to $\mathrm{mPFC}$ function. c-Fos and Npas4 are transcription factors that regulate downstream gene expression, and Arc encodes an effector protein that directly modulates cellular function [43]. c-Fos is expressed in an activity-dependent manner and is involved in complex learning and memory processes [23]. Arc is known to encode a synaptic protein that is involved in the generation of new synapses, and maintenance of the old synapses requires plasticity mechanisms (i.e., LTP) [23]. Npas4 plays an important role in adolescent maturation of the prefrontal inhibitory network [44] and regulates the excitatory and inhibitory balance at synaptic connections [45]. Thus, these IEGs are important for synaptic and neuronal plasticity, and they are considered involved in the development of psychiatric disorders (i.e., schizophrenia and autism) [23,46]. Prenatal MAM treatment did not alter the expression of any of the analysed IEGs in either sex. However, a gene-specific effect of adolescent JQ1 administration was observed. The effect of JQ1 on c-Fos expression was sex-specific, as it decreased the c-Fos mRNA level only in the male control and MAM groups. In contrast, adolescent inhibition of BET proteins 
induced an increase in Arc expression only in the MAM-treated group in both sexes. Npas4 transcription was also altered by JQ1 treatment, and similar to the regulation of $c$-Fos mRNA expression, a reduction in the level of the Npas4 mRNA was observed in male rats in the VEH and MAM groups. However, in females, a decrease in the Npas4 mRNA level was detected only in the control group. Nonetheless, correlation data showed a positive association between $c$-Fos and Npas4 expression in both sexes that might suggest an important role of both genes in JQ1's effect on neuronal activity in the mPFC. Recent evidence reported that JQ1 regulates c-Fos and Arc gene expression in vitro [12]. Thus, our results confirm previous findings related to c-Fos and Arc regulation, but also suggest that Npas4 transcription might be regulated by JQ1. The direction of changes in c-Fos and Npas4 expression observed in the present study were related to decreases in behavioural response (sensorimotor gating and novel object recognition) induced by JQ1 treatment in males. In females, only decreased levels of the Npas4 transcript in the MPFC might be related to an effect of JQ1 on the behavioural response of the control group (reduction in DI in the novel object recognition task and lack of effect on sensorimotor gating), as well as some slight effect on LTP observed in the female cortex. Thus, adolescent JQ1 administration modified neuronal and synaptic activity in the adult mPFC by altering expression of IEGs, and males might be more vulnerable to adolescent JQ1 treatment than females.

Our previous study showed a decrease in the mRNA levels of both GAD67 and PV induced by prenatal MAM treatment in the adult male $\mathrm{mPFC}[17,18,25]$. In the present study, we also observed a decrease in GAD67 and $P V$ expression in the $\mathrm{mPFC}$ of males but not females. Adolescent JQ1 treatment did not affect the expression of either GAD67 or PV in the mPFC of either sex. Thus, the dysfunction of GABAergic interneurons in the male $\mathrm{mPFC}$ might be involved in the deficits in sensorimotor gating, memory recognition, and LTP observed in MAM-treated males, but not female rats.

A correlation study of behavioural responses and gene expression also suggested sex-specific relationships in the association between these factors. Behavioural responses correlated with the expression of different genes in males (Gad1) and females (activitydependent genes: c-Fos and Npas4); however, Arc expression appears to be important in both sexes. The correlations between the analysed genes were also different in males and females, and the correlations between the expression of $B R D$ genes and IEGs were stronger in males than in females. The latter observation suggests that alterations in $B R D$ gene transcription might exert a stronger effect on IEG gene expression in males than in females. Therefore, adolescent treatment with JQ1 might exert a stronger effect on developmental abnormalities in males than females.

\subsection{Proteomic Alterations in the Adult mPFC}

Our results revealed functional and molecular changes in the adult mPFC of both sexes induced by both MAM and JQ1 administration, but the main changes were detected in males. Thus, we performed a proteomic study to investigate proteins that might be affected by either prenatal or postnatal treatment in both sexes.

Administration of either the prenatal risk factor MAM or JQ1 during adolescence altered protein levels in the male $\mathrm{MPFC}$, and based on the direction of changes, proteins were divided into 4 categories. The first category of proteins showed decreased expression in the MAM-CON group. Two are related to synapse function (NEUM and SYUB), and one is involved in energy transfer processes (ATPD). The second category is a large group of proteins showing increased levels in the JQ1 groups (VEH-JQ1 and MAM-JQ1). They are related to synapse function and signalling pathways (CNTN1, CALM, CTNB1, AP2B1, AT1A2, NCAM1, CPLX1, KPCG, TENR, SYN2, NSF, VGLU1, GNAO, and VDAC1), cytoskeletal organisation (CAZA2, MYPR, MTAP2, GDIA, and DPYL4), metabolic processes (GLNA, PGK1, and KPYM), and energy transfer processes (MDHC, SUCA, ADT1, and ADT2). The levels of proteins in the third category were decreased in the JQ1 groups (VEH-JQ1 and MAM-JQ1), and they are mainly involved in synapse function (DCKL1 and RAP1B), cytoskeletal organisation (DPYL2, GMFB, NCALD, and ARP3), and metabolic 
and energy transfer processes (ENOA, ATPB, and KCRU). Levels of proteins in the fourth category were increased mainly in the MAM-CON group, and they are related to synapse function and signalling processes (AP2A2, CLH1, GABT, and KAPCB) or metabolic and energy transfer processes (CISY, SDHA VATL, VATA, and NDUBA).

Proteins identified in the female $\mathrm{mPFC}$ were divided mainly into 2 categories based on the effects of the JQ1 treatment. Levels of proteins in the first category were decreased in both the VEH-JQ1 and MAM-JQ1 groups, and they are related to synapse function or cytoskeletal organisation (AT1A1, TENR, EAA1, CAND1, TBB4A, CLH1, and DCLK1) or are involved in metabolic and energy transfer processes (NDUV2 and TCPB). The levels of proteins in the second category increased in the VEH-JQ1 and MAM-JQ1 groups, and they are involved in synaptic (FKB1A, CNTN1, SYN1, and VDAC1) and cytoskeletal organisation (SPTN1, DPYL4, SYUB, and SPTN2), as well as metabolic (LDHC, ENOG, PGK1, KPYM, and HINT1) and energy transfer processes (EF1A, EFTU, COX5B, and VATG2).

Our previous proteomic study of the male adult mPFC showed a MAM-induced decrease in the levels of proteins related to synapse and cytoskeletal organisation and a MAM-evoked increase in the levels of proteins related to metabolic and energy transfer processes [47]. The present study also showed a decrease in the levels of synaptic proteins in MAM-treated males, which might suggest changes in neuropil reorganisation in the adult $\mathrm{mPFC}$ and might explain the reduction in the adult mPFC volume of MAM-E17 males reported in our previous study [18]. In female $\mathrm{mPFC}$, the levels of proteins related to synapse function and signalling pathways, as well as metabolic and energetic processes, were not changed after MAM treatment. The proteomic analysis identified $85 \%$ more altered proteins in MAM- and JQ1-treated males than in females, where only 15\% of proteins were altered. Thus, MAM administration mainly altered protein levels in the male mPFC. Sex differences in the proteomic landscape of the MAM-treated adult mPFC were also observed by other researchers [27]. A proteomic study performed in the human prefrontal cortex indicated alterations in proteins in patients with schizophrenia. These proteins are related to dysfunctional metabolism, cytoskeleton-related abnormalities, and cellular connections [48]. Thus, the proteins that were altered in our study might be involved in schizophrenia-like abnormalities observed mainly in male MAM-treated rats.

The robust effect of adolescent JQ1 treatment on protein levels in the MPFC of both VEH- and MAM-treated rats was observed in synaptic and cytoskeletal proteins, as well as proteins involved in metabolic and energy transfer processes, in both sexes. The levels of synaptic proteins were altered in both sexes (CLH1, CNTN1, DCLK1, SYUB, TENR, and VDAC1); however, the TENR protein was regulated in a sex-specific manner, and CLH1 and SYUB were regulated only in females by JQ1. Our results showing changes induced by JQ1 in synaptic proteins are consistent with RNA-sequencing data showing JQ1-induced alterations in the transcription of synaptic proteins and receptors in neurons [12].

\section{Materials and Methods}

\subsection{Animals and Treatment}

Pregnant dams (Wistar Han rats) were obtained from an animal provider (Charles River, Sulzfeld, Germany) when the foetuses were aged embryonic day 15 (E15) and housed individually in polycarbonate cages $(26.5 \times 18 \times 42 \mathrm{~cm})$. The rats were randomly assigned to experimental groups; at E17, the pregnant females were intraperitoneally (i.p.) injected with $22 \mathrm{mg} / \mathrm{kg}$ of methylazoxymethanol acetate (MAM, MRIGlobal, Kansas City, MO, USA) or saline vehicle $(0.9 \% \mathrm{NaCl})[7,17,18,35]$. The offspring were weaned 21 days after birth, and males and females were used in our experiments. The rats were housed in large polycarbonate boxes $(38 \times 20 \times 60 \mathrm{~cm})$ in groups of 4 in the same sex on an artificial 12/12 h light/dark cycle (lights on at 7 A.M.) with ad libitum access to food and water. The experimental groups consisted of animals chosen randomly from different litters (total 16) to avoid litter effects (maximum 2 rats from a single litter per group). The BET inhibitor (+)JQ1 ((6S)-4-(4-chlorophenyl)-2,3,9-trimethyl-6H-thieno[3,2- 
$f][1,2,4]$ triazolo[4,3-a][1,4]diazepine-6-acetic acid 1,1-dimethylethyl ester; STI, Poznan, Poland) was administered at a dose of $25 \mathrm{mg} / \mathrm{kg}$ i.p. once a day for 7 consecutive days in early adolescence (23rd-29th days of life). JQ1 was dissolved in 10\% DMSO and then diluted in corn oil. All experiments (behavioural and biochemical) were performed on four experimental groups (VEH-CON, VEH-JQ1, MAM-CON, and MAM-JQ1) using the same set of animals, but electrophysiological studies were performed using different sets of rats (Figure 11). The behavioural experiments were performed in adult rats as described below. Rats performed a novel object recognition test at P60 followed by an acoustic startle response test a week later. For the behavioural studies, each experimental group consisted of 12 rats per group. The animals were sacrificed a month after the last test, and brain tissue was stored until use in biochemical experiments. Biochemical studies (qRT-PCR and proteomics) were performed on the same mPFC samples obtained from adult rats at P110, and each experimental group consisted of 4-6 animals. A separate set of animals was used for the electrophysiological study performed on the mPFC of adult animals aged P60-P110, and each group consisted of 6-9 animals.

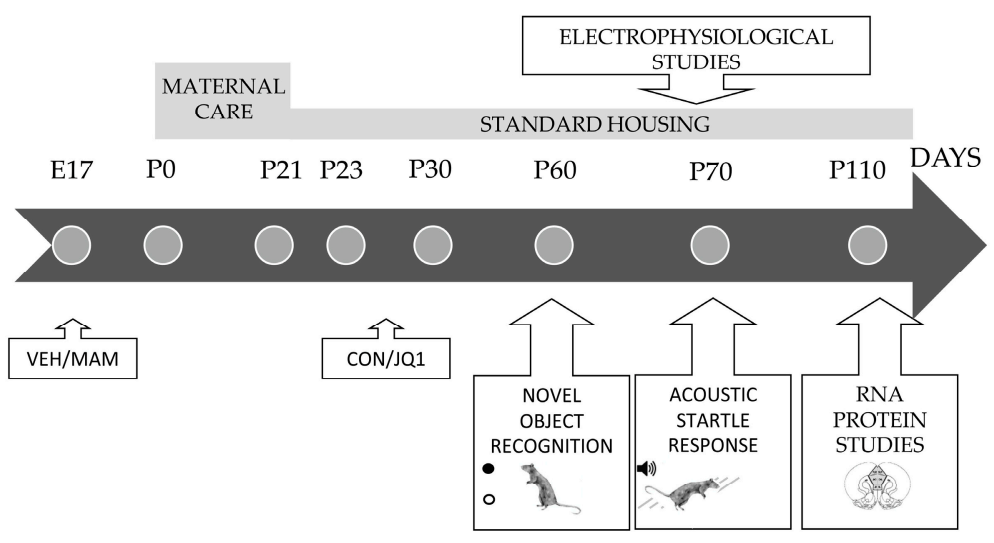

Figure 11. The schematic diagram depicts the adolescent administration of the inhibitor of BET proteins, JQ1 or vehicle (CON), of rats treated with vehicle (VEH) or MAM at embryonic day 17 (E17); P (postnatal days of life).

The study was carried out in strict accordance with the recommendations in the European Council Guide for the Care and Use of Laboratory Animals (86/609/EEC) and revised by Directive 2010/63/UE for the protection of animals used for scientific purposes. The protocols (permission number 138/2020; data of approval 4 June 2020) were approved by the Committee for Laboratory Animal Welfare and the Ethics of the Maj Institute of Pharmacology, Polish Academy of Sciences in Kraków, and were conducted in accordance with the current version of the Polish Law on the Protection of Animals (266/2015).

\subsection{Behavioural Study}

\subsubsection{Novel Object Recognition Test}

The study was performed as described previously with some modifications $[17,35]$. Rats were tested for memory deficits in dimly lit (18 lx) cages made of black Plexiglas $(60 \times 60 \times 30 \mathrm{~cm})$. After each measurement, the floor and walls were cleaned and dried. Twenty-four hours before training, the animals were habituated to the arena (without any objects) for $10 \mathrm{~min}$. Objects used for the discrimination task included a black glass cylinder and a white plastic cuboid filled with water. The objects were placed in two opposite corners of the arena, approximately $10 \mathrm{~cm}$ from the walls. The rats were placed in the middle of the arena, and during the training session (acquisition), two identical objects (A and A1) were presented for $5 \mathrm{~min}$ (half of the animals were presented with two black glass cylinders, and the other half with two white plastic cuboids). After a $1 \mathrm{~h}$ rest in the home cage, the rats were again placed in the same Plexiglas cage as in the previous test and presented with two objects for $3 \mathrm{~min}$, but one of the objects was replaced by a novel object 
(A-familiar and B-novel) (recognition session). The objects were cleaned with a 70\% ethanol solution between sessions. Exploration was defined as sniffing the object and/or touching it with the nose or forepaws. The animal behaviour was automatically recorded by cameras (SJ8Pro SJCAM, China) and next, videos were analysed using the Solomon Coder program (https:// solomon.andraspeter.com, accessed on 5 August 2021). Animals showing less than $5 \mathrm{~s}$ of exploration time were excluded from the experiment. Based on the time spent exploring $(\mathrm{E})$ each object, a discrimination index $(\mathrm{DI})$ was calculated: $\mathrm{DI}=\left(\mathrm{E}_{\mathrm{B}}\right.$ $\left.-E_{A}\right) /\left(E_{A}+E_{B}\right)$.

\subsubsection{Acoustic Startle Response Test}

The efficacy of sensorimotor gating was measured by recording pre-pulse inhibition (PPI) of the acoustic startle response, and reactivity was measured using a startle apparatus (SR-LAB, San Diego Instruments, San Diego, CA, USA), as described previously [7,17,18,35]. Briefly, after a 5 min habituation period that included playing background white noise at $65 \mathrm{~dB}$, two types of acoustic stimuli were presented in a random order: an acoustic stimulus alone $((\mathrm{P})$, duration $40 \mathrm{~ms}$, intensity $120 \mathrm{~dB})$ and an acoustic stimulus preceded by an acoustic pre-pulse ((PP), duration $20 \mathrm{~ms}$, intensities: 70, 75, and 80 ) applied $80 \mathrm{~ms}$ before the stimulus (P). During each experimental session, 20 trials of each type were presented with randomised interstimulus intervals ranging from 5 to $20 \mathrm{~s}$ in length. The amplitudes were averaged separately for each animal and for both types of trials (stimulus alone, $\mathrm{P}$; stimulus preceded by the pre-pulse, PP). The degree of pre-pulse inhibition was calculated as the percentage of inhibition $(\% \mathrm{PPI})$ using the following formula: $[(\mathrm{P}-\mathrm{PP}) / \mathrm{P}] \times 100$.

\subsection{Electrophysiological Study}

The electrophysiological procedure has been described previously [49]. The procedure was performed on adult rats between P60 and P110. The rats from each experimental group were tested in a counterbalanced, alternating order. Rats were anesthetised with isoflurane (Aerrane, Baxter, Warsaw, Poland) and decapitated. The brain was rapidly removed and immersed in an ice-cold artificial cerebrospinal fluid (ACSF) of the following composition (in $\mathrm{mM}$ ): $130 \mathrm{NaCl}, 5 \mathrm{KCl}, 2.5 \mathrm{CaCl}_{2}, 1.3 \mathrm{MgSO}_{4}, 1.25 \mathrm{NaH}_{2} \mathrm{PO}_{4}, 26 \mathrm{NaHCO}_{3}$, and 10 d-glucose, and ACSF was bubbled with a mixture of $95 \% \mathrm{O}_{2}$ and $5 \% \mathrm{CO}_{2}(\mathrm{pH}$ 7.4). A block of tissue containing the mPFC was dissected and cut into $410 \mu \mathrm{m}$ thick sections along the coronal plane using a vibratome at $1^{\circ} \mathrm{C}$ (VT 1000S, Leica, Germany). The sections were transferred into a recording chamber with a fluid-gas interface and superfused at ate of $2 \mathrm{~mL} / \mathrm{min}$ with warm $\left(32 \pm 0.5^{\circ} \mathrm{C}\right.$ )-modified ACSF containing (in $\mathrm{mM}$ ): $130 \mathrm{NaCl}, 2$ $\mathrm{KCl}, 2.5 \mathrm{CaCl}_{2}, 1.3 \mathrm{MgSO}_{4}, 1.25 \mathrm{NaH}_{2} \mathrm{PO}_{4}, 26 \mathrm{NaHCO}_{3}$, and $10 \mathrm{D}$-glucose. The recordings started 2-4 h after the sections were prepared. The field potentials were evoked using square-wave stimuli (duration $0.2 \mathrm{~ms}$ ) applied by a constant current isolation unit (WPI, Germany) at $0.016 \mathrm{~Hz}$ via a bipolar concentric Pt-Ir electrode (FHC, Bowdoin, ME, USA). The electrode was placed in layer II/III of the MPFC. A recording micropipette filled with ACSF (resistance of approximately 2-4 M $\Omega$ ) was placed in cortical layer $V$ of the mPFC. The responses were amplified $(500 \times)$, filtered $(0.1 \mathrm{~Hz}-1.3 \mathrm{kHz})$, and acquired at a $10 \mathrm{kHz}$ sampling rate (EXT 10-C amplifier, NPI, Germany) using a Micro1401 interface and Signal 2 software (CED, Cambridge, UK).

After stabilisation of the responses, stimulus/response (input-output) curves were determined individually for each section by applying square stimuli of increasing intensities (duration of $0.2 \mathrm{~ms}$; range of $0-50 \mu \mathrm{A}$, step $5 \mu \mathrm{A}$; range of $50-100 \mu \mathrm{A}$, step $10 \mu \mathrm{A}$; applied at $15 \mathrm{~s}$ intervals). Then, the stimulation intensity was adjusted to evoke a response corresponding to $40 \%$ of the maximum amplitude. When the signals became stable over a baseline period of $15 \mathrm{~min}$, LTP was induced in the $\mathrm{mPFC}$ with a sequence of five stimulus bursts (15 s apart). A single burst consisted of 5 individual square pulses (duration of $0.3 \mathrm{~ms}$, applied at $100 \mathrm{~Hz}$ ) that were repeated ten times ( $0.2 \mathrm{~s}$ apart). The recording then continued for an additional $90 \mathrm{~min}$. The response amplitude of the individual sections was normalised to the percent change relative to the baseline values. The amount of LTP was 
determined by comparing the data from the baseline recording averaged over the last 15 $\mathrm{min}$ to the data from the post-tetanic recording averaged over the last $15 \mathrm{~min}$.

\subsection{Biochemical Study}

\subsubsection{Tissue Preparation}

After the rats were decapitated, the brains were removed, cooled on ice, and sliced into $1 \mathrm{~mm}$ coronal sections using a rodent brain matrix (Ted Pella, Inc., Redding, CA, USA). The $\mathrm{mPFC}$ was dissected from coronal sections, frozen in liquid nitrogen, and stored at $-20^{\circ} \mathrm{C}$. Simultaneous extraction of proteins and nucleic acids from the same experimental sample was performed using commercially available kits (Nucleospin RNA/Protein, MachereyNagel, RNAeasy, Qiagen, Wroclaw, Poland) and columns (Amicon Ultra, Millipore, Warsaw, Poland) with some modifications. RNA was extracted using the RNeasy kit according to the manufacturer's instructions (www1.qiagen.com/literature/handbooks, accessed on 30 June 2021). The flow through was collected, and proteins were extracted using a Nucleospin RNA/Protein kit according to the manufacturer's instruction (www.mn-net.com/media/ pdf, accessed on 30 June 2021) and purified with Amicon Ultra Centrifugal Filter Units (Amicon). Proteins were dissolved in Dissociation Buffer (SCIEX) and quantified using the bicinchoninic acid (BCA) Protein Assay kit (Pierce, Rockford, IL, USA) according to the manufacturer's instructions (www.piercenet.com/files/1296dh4.pdf, accessed on 30 June 2021).

The obtained mRNA and proteins were used for qRT-PCR or proteomic studies, respectively.

\subsection{2. qRT-PCR Study}

qRT-PCR was performed using the QuantStudio 12K Flex Real-Time PCR System (Applied Biosystems, Warsaw, Poland) as previously described [25]. The gene-specific primers and probes (see details in Table 3) from the TaqMan Gene Expression Assays (Applied Biosystems) were used, and $1 \mu \mathrm{L}$ of each sample was used for cDNA amplification. Amplification was performed using the TaqMan Universal PCR Master Mix (Applied Biosystems) under the following conditions: $50^{\circ} \mathrm{C}$ for $2 \mathrm{~min}$ and $95^{\circ} \mathrm{C}$ for $10 \mathrm{~min}$, followed by 40 cycles of $95^{\circ} \mathrm{C}$ for $15 \mathrm{~s}$ and $60^{\circ} \mathrm{C}$ for $1 \mathrm{~min}$. The expression of the glyceraldehyde3-phosphate dehydrogenase (Gapdh) transcript (assay Rn99999916_s1) was quantified to control for variations in cDNA levels. All qRT-PCR experiments were performed in duplicate and included no-template controls. The cycle threshold values were calculated automatically. Relative quantification was performed using the comparative threshold (CT) method, $2-\Delta \mathrm{CT}$, where $\Delta \mathrm{CT}=(\mathrm{CT}$, target gene $-\mathrm{CT}$, reference gene $)$.

Table 3. A list of a gene-specific primers and probes.

\begin{tabular}{ccc}
\hline Assay ID & Gene Symbol & Gene Name \\
\hline Rn99999916_s1 & GAPDH & $\begin{array}{c}\text { glyceraldehyde-3-phosphate } \\
\text { dehydrogenase }\end{array}$ \\
Rn01435739_m1 & BRD3 & bromodomain containing 3 \\
Rn01428703_m1 & BRD2 & bromodomain containing 2 \\
Rn01535560_m1 & BRD4 & bromodomain containing 4 \\
Rn00574541_m1 & Pvalb & parvalbumin \\
Rn00566593_m1 & Gad1 & glutamate decarboxylase 1 \\
Rn02396759_m1 & Fos & FBJosteosarcoma oncogene \\
Rn01454622_g1 & Npas4 & neuronal PASdomain protein 4 \\
Rn00571208_g1 & Arc & Activity-regulated cytoskeletal \\
\hline
\end{tabular}




\subsubsection{Proteomic Analysis}

\section{- Protein Extraction}

The study was performed as described previously with some modifications [47]. Protein concentrations of the obtained fraction were determined using a BCA protein assay (Sigma-Aldrich, Poznan, Poland). One hundred micrograms of protein from each sample were used for analysis. Afterwards, samples were reduced by Tris-(2-carboxyethyl)phosphine (TCEP, Sigma-Aldrich, and cysteines were blocked according to the iTRAQ protocol (AB Sciex, Framingham, MA, USA). Proteins were digested using Sequencing Grade Modified Trypsin (from Promega, Warsaw, Poland) overnight at $37^{\circ} \mathrm{C}$.

- Stable isotope labelling

Samples were labelled using iTRAQ reagents (AB Sciex, Framingham, MA, USA), as recommended by the manufacturer for 8-plex iTRAQ. Labelled samples were combined and dried in a vacuum centrifuge (Labconco, VWR, Gdansk, Poland).

- Strong cation-exchange fractionation

Lyophilisates were dissolved in $10 \mathrm{mM}$ of potassium phosphate containing $25 \%$ acetonitrile at $\mathrm{pH}$ 3.0. The samples were purified on an Applied Biosystems Cation-Exchange Cartridge. The peptides were eluted from the column with $10 \mathrm{mM}$ of potassium phosphate containing $25 \%$ acetonitrile at $\mathrm{pH} 3.0$ and $350 \mathrm{mM}$ of potassium chloride (Sigma-Aldrich). The eluate was dried in a vacuum centrifuge, reconstituted in $2 \%$ acetonitrile and $0.1 \%$ formic acid, and subjected to MacroSpin Columns with C18 resin (the Nest Group, Inc., Ipswich, MA, USA) to remove salts. The samples were eluted with $80 \%$ acetonitrile containing $0.1 \%$ formic acid, dried in a vacuum centrifuge again, and redissolved in $2 \%$ acetonitrile containing $0.1 \%$ formic acid for nano-LC-MALDI analyses.

- Chromatographic separation and spotting

Chromatographic separation of peptides was achieved on a Proxeon EASY-nLC system (Bruker Daltonics, Bremen, Germany) equipped with a $2 \mathrm{~cm}$ long, $100 \mu \mathrm{m}$ ID precolumn containing $5 \mu \mathrm{m}$ BioSphere C18 resin (NanoSeparations, Nieuwkoop, The Netherlands) and a $10 \mathrm{~cm}$ long, $75 \mu \mathrm{m}$ ID fused silica column containing $3 \mu \mathrm{m}$ BioSphere $\mathrm{C} 18$ resin (NanoSeparations, The Netherlands). The gradient was formed using $0.1 \%$ formic acid in water (solvent $\mathrm{A}$ ) and $0.1 \%$ formic acid in acetonitrile (solvent $\mathrm{B}$ ). The system was controlled by Hystar software (Bruker Daltonics). A gradient was established from $2 \%$ to $35 \% \mathrm{~B}$ in $50 \mathrm{~min}$, that increased to $90 \% \mathrm{~B}$ at $55 \mathrm{~min}$ and then was maintained until 65 min. Samples were loaded from a cooled $\left(7^{\circ} \mathrm{C}\right)$ autosampler and separated with a linear gradient that was formed at a flow rate of $300 \mathrm{nl} / \mathrm{min}$. Chromatographic separation was controlled by the Hystar system (Bruker Daltonics). The LC eluent was directly mixed with $\alpha$-cyano-4-hydroxycinnamic acid matrix (HCCA, Bruker Daltonics) and deposited on an MTP AnchorChip 1536 BC plate (Bruker, Daltonik). The LC eluent was spotted with a Proteineer fc II (Bruker, Daltonik). Spots were deposited every 15 s, and 192 fractions were spotted in total for one sample.

\section{- $\quad$ LC-MALDI-TOF/TOF analysis}

All data were acquired on an ultrafleXtreme (BrukerDaltonik, Bremen, Germany) mass spectrometer with a TOF/TOF analyser. All MS spectra were recorded in positive reflector mode (700-3500 Da). For MS data, 4000 shots were accumulated for each spectrum. Collected data were analysed using WARP-LC software (Bruker Daltonics, Bremen, Germany). Search parameters were set as follows: taxonomy: Rodentia (UniProt/Swiss-Prot, Expasy, Swiss Institute of Bioinformatics (www.expasy.org, accessed on 11 August 2021); enzyme: trypsin; missed cleavage sites allowed: 1 ; fixed modification: methylation $(\mathrm{C})$; variable modifications: methionine oxidation (M), iTRAQ8plex (K), iTRAQ8plex (N-term), and iTRAQ8plex (Y); parent mass error: 50 ppm; peptide fragment mass tolerance: $0.6 \mathrm{Da}$. 


\section{- Protein interaction network analysis}

Differentially expressed proteins identified in the present study were subjected to a pathway analysis. For this purpose, the Swiss-Prot accession numbers were inserted into STRING (Search Tool for the Retrieval of Interacting Genes/Proteins) software (http:/ / string.embl.de, accessed on 15 June 2021).

- Database searching

In addition, all the identified proteins were searched using UniProtKB and the Panther Database to establish their molecular functions and interactions.

- Proteomic data depository

The mass spectrometry proteomics data have been deposited to the ProteomeXchange Consortium via the PRIDE partner repository with the dataset identifiers PXD027050 and 10.6019/PXD027050.

\subsection{Statistical Analysis}

The results are presented as the group means \pm the standard errors of the means (SEM). Statistical evaluations were performed using Statistica software and consisted of one-way or two-way repeated measures analyses of variance (ANOVAs), as indicated in the figures, followed by the Tukey post hoc test. The between- (prenatal and postnatal treatment) and within-subjects (pre-pulse intensity for the acoustic startle response test or objects for the novel object recognition test) factors were used for the ANOVAs. Differences were considered significant at $p$ textless 0.05 .

All statistical analyses of proteomics data were performed using $\mathrm{R}$ software version 3.6.2. Corrections for multiple testing were performed using Bonferroni's method. Proteomic data were subjected to two-way ANOVA with prenatal or postnatal treatment as factors, followed by correction for multiple testing using Bonferroni's method. Differences were considered significant at $p$ textless 0.05 .

Associations among gene expression and proteomic and behavioural data were estimated by calculating Pearson's rank-based correlation coefficients, followed by correction for multiple testing using the Benjamini-Hochberg method to decrease the false discovery rate.

\section{Conclusions}

In our study, a prenatal MAM treatment exerted a sex-specific effect on the development of schizophrenia-like abnormalities, and only males were affected. Adolescent administration of JQ1 altered behavioural responses in control animals, mainly in males, but not in rats of both sexes in MAM-treated groups. Deficits in LTP in the mPFC were only observed in male MAM-treated animals, and adolescent JQ1 treatment did not affect cellular memory in either sex. The results from the electrophysiological study are consistent with proteomic data showing changes in synaptic proteins only in male MAM-treated rats. On the other hand, protein alterations induced by JQ1 did not affect cortical LTP. Thus, prenatal MAM treatment might change the responsiveness to BET protein functions during adolescence.

JQ1 treatment during adolescence might affect the direction of prefrontal cortical development in both sexes by modulating gene expression and the protein landscape. Although there are some limitations, and caution is required in comparison animal and human studies, the aforementioned results might be important not only for determining the emergence of schizophrenia but also for the administration of JQ1 therapy to this age group, which might be practised in children who are oncology patients [50].

Author Contributions: Conceptualisation, M.M. and W.B.; methodology, W.B., A.W.-B., I.M.-M., M.C. and M.M.; Investigation, W.B., A.W.-B., I.M.-M., M.C. and M.M.; formal analysis, W.B., I.M.-M. and M.M.; data curation, W.B.; writing —original draft preparation, M.M.; writing—review and editing, M.M. and W.B.; visualisation, W.B., I.M.-M. and M.M.; supervision, M.M.; project administration, 
M.M.; funding acquisition, M.M. All authors have read and agreed to the published version of the manuscript.

Funding: This research was funded by the National Science Centre, Poland, grant 2018/31/B/NZ4/01465.

Institutional Review Board Statement: The study was conducted according to the guidelines of the Declaration of Helsinki and approved by the Committee for Laboratory Animal Welfare and the Ethics of the Maj Institute of Pharmacology, Polish Academy of Sciences in Kraków, and was conducted in accordance with the current version of the Polish Law on the Protection of Animals (266/2015) (permission number 138/2020; data of approval 4 June 2020).

Informed Consent Statement: Not applicable.

Data Availability Statement: The mass spectrometry proteomics data have been deposited to the ProteomeXchange Consortium via the PRIDE partner repository with the dataset identifiers PXD027050 and 10.6019/PXD027050.

Acknowledgments: The proteomic research was performed in the Laboratory of Proteomics and Mass Spectrometry, Maj Institute of Pharmacology, Polish Academy of Sciences in Krakow, Poland.

Conflicts of Interest: The authors declare no conflict of interest.

\section{References}

1. McCutcheon, R.A.; Marques, T.R.; Howes, O.D. Schizophrenia-An Overview. JAMA Psychiatry 2020, 77, 201-210. [CrossRef]

2. Selemon, L.D.; Zecevic, N. Schizophrenia: A tale of two critical periods for prefrontal cortical development. Transl. Psychiatry 2015, 5, e623. [CrossRef] [PubMed]

3. Millan, M.J.; Andrieux, A.; Bartzokis, G.; Cadenhead, K.; Dazzan, P.; Fusar-Poli, P.; Gallinat, J.; Giedd, J.; Grayson, D.R.; Heinrichs, M.; et al. Altering the course of schizophrenia: Progress and perspectives. Nat. Rev. Drug Discov. 2016, 15, 485-515. [CrossRef]

4. Sommer, I.E.; Bearden, C.E.; van Dellen, E.; Breetvelt, E.J.; Duijff, S.N.; Maijer, K.; van Amelsvoort, T.; de Haan, L.; Gur, R.E.; Arango, C.; et al. Early interventions in risk groups for schizophrenia: What are we waiting for? NPJ Schizophr. 2016, 2, 16003. [CrossRef] [PubMed]

5. Millan, M.J. An epigenetic framework for neurodevelopmental disorders: From pathogenesis to potential therapy. Neuropharmacology 2013, 68, 2-82. [CrossRef] [PubMed]

6. Morrison, K.E.; Rodgers, A.B.; Morgan, C.P.; Bale, T.L. Epigenetic mechanisms in pubertal brain maturation. Neuroscience 2014, 264, 17-24. [CrossRef]

7. Bator, E.; Latusz, J.; Radaszkiewicz, A.; Wedzony, K.; Mackowiak, M. Valproic acid (VPA) reduces sensorimotor gating deficits and HDAC2 overexpression in the MAM animal model of schizophrenia. Pharmacol. Rep. 2015, 67, 1124-1129. [CrossRef]

8. Koseki, T.; Mouri, A.; Mamiya, T.; Aoyama, Y.; Toriumi, K.; Suzuki, S.; Nakajima, A.; Yamada, T.; Nagai, T.; Nabeshima, T. Exposure to enriched environments during adolescence prevents abnormal behaviours associated with histone deacetylation in phencyclidine-treated mice. Int. J. Neuropsychopharmacol. 2012, 15, 1489-1501. [CrossRef] [PubMed]

9. Prinjha, R.K.; Witherington, J.; Lee, K. Place your BETs: The therapeutic potential of bromodomains. Trends Pharmacol. Sci. 2012, 33, 146-153. [CrossRef]

10. Filippakopoulos, P.; Knapp, S. Targeting bromodomains: Epigenetic readers of lysine acetylation. Nat. Rev. Drug Discov. 2014, 13, 337-356. [CrossRef] [PubMed]

11. Singh, M.B.; Sartor, G.C. BET bromodomains as novel epigenetic targets for brain health and disease. Neuropharmacology 2020, 181, 108306. [CrossRef] [PubMed]

12. Korb, E.; Herre, M.; Zucker-Scharff, I.; Darnell, R.B.; Allis, C.D. BET protein Brd4 activates transcription in neurons and BET inhibitor Jq1 blocks memory in mice. Nat. Neurosci. 2015, 18, 1464-1473. [CrossRef]

13. Korb, E.; Herre, M.; Zucker-Scharff, I.; Gresack, J.; Allis, C.D.; Darnell, R.B. Excess Translation of Epigenetic Regulators Contributes to Fragile X Syndrome and Is Alleviated by Brd4 Inhibition. Cell 2017, 170, 1209-1223.e1220. [CrossRef] [PubMed]

14. Li, J.; Zhao, G.; Gao, X. Development of neurodevelopmental disorders: A regulatory mechanism involving bromodomaincontaining proteins. J. Neurodev. Disord. 2013, 5, 4. [CrossRef]

15. Sullivan, J.M.; Badimon, A.; Schaefer, U.; Ayata, P.; Gray, J.; Chung, C.W.; von Schimmelmann, M.; Zhang, F.; Garton, N.; Smithers, N.; et al. Autism-like syndrome is induced by pharmacological suppression of BET proteins in young mice. J. Exp. Med. 2015, 212, 1771-1781. [CrossRef]

16. Modinos, G.; Allen, P.; Grace, A.A.; McGuire, P. Translating the MAM model of psychosis to humans. Trends Neurosci. 2015, 38, 129-138. [CrossRef] [PubMed]

17. Bator, E.; Latusz, J.; Wedzony, K.; Mackowiak, M. Adolescent environmental enrichment prevents the emergence of schizophrenialike abnormalities in a neurodevelopmental model of schizophrenia. Eur. Neuropsychopharmacol. 2018, 28, 97-108. [CrossRef]

18. Mackowiak, M.; Bator, E.; Latusz, J.; Mordalska, P.; Wedzony, K. Prenatal MAM administration affects histone H3 methylation in postnatal life in the rat medial prefrontal cortex. Eur. Neuropsychopharmacol. 2014, 24, 271-289. [CrossRef] 
19. Geyer, M.A. The family of sensorimotor gating disorders: Comorbidities or diagnostic overlaps? Neurotox. Res. 2006, 10, 211-220. [CrossRef]

20. Swerdlow, N.R.; Weber, M.; Qu, Y.; Light, G.A.; Braff, D.L. Realistic expectations of prepulse inhibition in translational models for schizophrenia research. Psychopharmacology 2008, 199, 331-388. [CrossRef]

21. Lyon, L.; Saksida, L.M.; Bussey, T.J. Spontaneous object recognition and its relevance to schizophrenia: A review of findings from pharmacological, genetic, lesion and developmental rodent models. Psychopharmacology 2012, 220, 647-672. [CrossRef] [PubMed]

22. Rajagopal, L.; Massey, B.W.; Huang, M.; Oyamada, Y.; Meltzer, H.Y. The novel object recognition test in rodents in relation to cognitive impairment in schizophrenia. Curr. Pharm. Des. 2014, 20, 5104-5114. [CrossRef]

23. Gallo, F.T.; Katche, C.; Morici, J.F.; Medina, J.H.; Weisstaub, N.V.; Morici, J.F. Immediate Early Genes, Memory and Psychiatric Disorders: Focus on c-Fos, Egr1 and Arc. Front. Behav. Neurosci. 2018, 12, 79. [CrossRef]

24. Schmidt, M.J.; Mirnics, K. Neurodevelopment, GABA system dysfunction, and schizophrenia. Neuropsychopharmacology 2015, 40, 190-206. [CrossRef]

25. Mackowiak, M.; Latusz, J.; Glowacka, U.; Bator, E.; Bilecki, W. Adolescent social isolation affects parvalbumin expression in the medial prefrontal cortex in the MAM-E17 model of schizophrenia. Metab. Brain Dis. 2019, 34, 341-352. [CrossRef]

26. Canuso, C.M.; Pandina, G. Gender and schizophrenia. Psychopharmacol. Bull. 2007, 40, 178-190.

27. Chalkiadaki, K.; Velli, A.; Kyriazidis, E.; Stavroulaki, V.; Vouvoutsis, V.; Chatzaki, E.; Aivaliotis, M.; Sidiropoulou, K. Development of the MAM model of schizophrenia in mice: Sex similarities and differences of hippocampal and prefrontal cortical function. Neuropharmacology 2019, 144, 193-207. [CrossRef]

28. Huo, C.; Liu, X.; Zhao, J.; Zhao, T.; Huang, H.; Ye, H. Abnormalities in behaviour, histology and prefrontal cortical gene expression profiles relevant to schizophrenia in embryonic day 17 MAM-Exposed C57BL/6 mice. Neuropharmacology 2018, 140, 287-301. [CrossRef] [PubMed]

29. Potasiewicz, A.; Holuj, M.; Litwa, E.; Gzielo, K.; Socha, L.; Popik, P.; Nikiforuk, A. Social dysfunction in the neurodevelopmental model of schizophrenia in male and female rats: Behavioural and biochemical studies. Neuropharmacology 2020, 170, 108040. [CrossRef]

30. Flagstad, P.; Glenthoj, B.Y.; Didriksen, M. Cognitive deficits caused by late gestational disruption of neurogenesis in rats: A preclinical model of schizophrenia. Neuropsychopharmacology 2005, 30, 250-260. [CrossRef] [PubMed]

31. Flagstad, P.; Mork, A.; Glenthoj, B.Y.; van Beek, J.; Michael-Titus, A.T.; Didriksen, M. Disruption of neurogenesis on gestational day 17 in the rat causes behavioral changes relevant to positive and negative schizophrenia symptoms and alters amphetamineinduced dopamine release in nucleus accumbens. Neuropsychopharmacology 2004, 29, 2052-2064. [CrossRef]

32. Le Pen, G.; Gourevitch, R.; Hazane, F.; Hoareau, C.; Jay, T.M.; Krebs, M.O. Peri-pubertal maturation after developmental disturbance: A model for psychosis onset in the rat. Neuroscience 2006, 143, 395-405. [CrossRef]

33. Moore, H.; Jentsch, J.D.; Ghajarnia, M.; Geyer, M.A.; Grace, A.A. A neurobehavioral systems analysis of adult rats exposed to methylazoxymethanol acetate on E17: Implications for the neuropathology of schizophrenia. Biol. Psychiatry 2006, 60, 253-264. [CrossRef]

34. Millan, M.J.; Agid, Y.; Brune, M.; Bullmore, E.T.; Carter, C.S.; Clayton, N.S.; Connor, R.; Davis, S.; Deakin, B.; DeRubeis, R.J.; et al. Cognitive dysfunction in psychiatric disorders: Characteristics, causes and the quest for improved therapy. Nat. Rev. Drug Discov. 2012, 11, 141-168. [CrossRef]

35. Bator, E.; Latusz, J.; Glowacka, U.; Radaszkiewicz, A.; Mudlaff, K.; Mackowiak, M. Adolescent Social Isolation Affects Schizophrenia-Like Behavior in the MAM-E17 Model of Schizophrenia. Neurotox. Res. 2018, 34, 305-323. [CrossRef] [PubMed]

36. Perez, S.M.; Donegan, J.J.; Lodge, D.J. Effect of estrous cycle on schizophrenia-like behaviors in MAM exposed rats. Behav. Brain Res. 2019, 362, 258-265. [CrossRef]

37. Kumari, V.; Aasen, I.; Sharma, T. Sex differences in prepulse inhibition deficits in chronic schizophrenia. Schizophr. Res. 2004, 69, 219-235. [CrossRef]

38. Rahman, Q.; Kumari, V.; Wilson, G.D. Sexual orientation-related differences in prepulse inhibition of the human startle response. Behav. Neurosci. 2003, 117, 1096-1102. [CrossRef] [PubMed]

39. Braff, D.L.; Light, G.A.; Ellwanger, J.; Sprock, J.; Swerdlow, N.R. Female schizophrenia patients have prepulse inhibition deficits. Biol. Psychiatry 2005, 57, 817-820. [CrossRef] [PubMed]

40. Sartor, G.C.; Malvezzi, A.M.; Kumar, A.; Andrade, N.S.; Wiedner, H.J.; Vilca, S.J.; Janczura, K.J.; Bagheri, A.; Al-Ali, H.; Powell, S.K.; et al. Enhancement of BDNF Expression and Memory by HDAC Inhibition Requires BET Bromodomain Reader Proteins. $J$ Neurosci. 2019, 39, 612-626. [CrossRef]

41. Shaw, G.A.; Dupree, J.L.; Neigh, G.N. Adolescent maturation of the prefrontal cortex: Role of stress and sex in shaping adult risk for compromise. Genes Brain Behav. 2020, 19, e12626. [CrossRef] [PubMed]

42. Benito, E.; Ramachandran, B.; Schroeder, H.; Schmidt, G.; Urbanke, H.; Burkhardt, S.; Capece, V.; Dean, C.; Fischer, A. The BET/BRD inhibitor JQ1 improves brain plasticity in WT and APP mice. Transl. Psychiatry 2017, 7, e1239. [CrossRef]

43. Sun, X.; Lin, Y. Npas4: Linking Neuronal Activity to Memory. Trends Neurosci. 2016, 39, 264-275. [CrossRef]

44. Shepard, R.; Heslin, K.; Coutellier, L. The transcription factor Npas4 contributes to adolescent development of prefrontal inhibitory circuits, and to cognitive and emotional functions: Implications for neuropsychiatric disorders. Neurobiol. Dis. 2017, 99, 36-46. [CrossRef] 
45. Spiegel, I.; Mardinly, A.R.; Gabel, H.W.; Bazinet, J.E.; Couch, C.H.; Tzeng, C.P.; Harmin, D.A.; Greenberg, M.E. Npas4 regulates excitatory-inhibitory balance within neural circuits through cell-type-specific gene programs. Cell 2014, 157, 1216-1229. [CrossRef] [PubMed]

46. Coutellier, L.; Beraki, S.; Ardestani, P.M.; Saw, N.L.; Shamloo, M. Npas4: A neuronal transcription factor with a key role in social and cognitive functions relevant to developmental disorders. PLoS ONE 2012, 7, e46604. [CrossRef]

47. Bilecki, W.; Latusz, J.; Gawlinska, K.; Chmelova, M.; Mackowiak, M. Prenatal MAM treatment altered fear conditioning following social isolation: Relevance to schizophrenia. Behav. Brain Res. 2021, 406, 113231. [CrossRef]

48. Martins-de-Souza, D.; Gattaz, W.F.; Schmitt, A.; Maccarrone, G.; Hunyadi-Gulyas, E.; Eberlin, M.N.; Souza, G.H.; Marangoni, S.; Novello, J.C.; Turck, C.W.; et al. Proteomic analysis of dorsolateral prefrontal cortex indicates the involvement of cytoskeleton, oligodendrocyte, energy metabolism and new potential markers in schizophrenia. J. Psychiatr. Res. 2009, 43, 978-986. [CrossRef]

49. Solarz, A.; Majcher-Maslanka, I.; Kryst, J.; Chocyk, A. A Search for Biomarkers of Early-life Stress-related Psychopathology: Focus on 70-kDa Heat Shock Proteins. Neuroscience 2021, 463, 238-253. [CrossRef]

50. Jimenez, I.; Baruchel, A.; Doz, F.; Schulte, J. Bromodomain and extraterminal protein inhibitors in pediatrics: A review of the literature. Pediatr. Blood Cancer 2017, 64, e26334. [CrossRef] [PubMed] 